\title{
Sensitivity of Noisy Neurons to Coincident Inputs
}

\author{
Cyrille Rossant, ${ }^{1,2}$ Sara Leijon, ${ }^{3,4}$ Anna K. Magnusson, ${ }^{3,4}$ and Romain Brette ${ }^{1,2}$ \\ ${ }^{1}$ Laboratoire Psychologie de la Perception, CNRS, Université Paris Descartes, F-75006 Paris, France, ${ }^{2}$ Equipe Audition, Département d’Etudes Cognitives, \\ Ecole Normale Supérieure, 75005 Paris, France, and ${ }^{3}$ Center for Hearing and Communication Research and ${ }^{4}$ Department of Clinical Science, Intervention \\ and Technology, Karolinska Institutet, 17176 Stockholm, Sweden
}

How do neurons compute? Two main theories compete: neurons could temporally integrate noisy inputs (rate-based theories) or they could detect coincident input spikes (spike timing-based theories). Correlations at fine timescales have been observed in many areas of the nervous system, but they might have a minor impact. To address this issue, we used a probabilistic approach to quantify the impact of coincidences on neuronal response in the presence of fluctuating synaptic activity. We found that when excitation and inhibition are balanced, as in the sensory cortex in vivo, synchrony in a very small proportion of inputs results in dramatic increases in output firing rate. Our theory was experimentally validated with in vitro recordings of cortical neurons of mice. We conclude that not only are noisy neurons well equipped to detect coincidences, but they are so sensitive to fine correlations that a rate-based description of neural computation is unlikely to be accurate in general.

\section{Introduction}

What is the role of precise spike timing in neural computation? This fundamental issue remains highly controversial. The traditional view postulates that neurons temporally integrate noisy inputs, with precise spike timing playing little role in neural function (Adrian, 1934; Shadlen and Newsome, 1998; London et al., 2010): the output firing rate is essentially determined by the input firing rates. In the last decades, a number of authors have advocated radically different views, in which the temporal coordination of spikes plays a central role (Singer, 1999; VanRullen et al., 2005). A popular spike-timing theory proposes that neurons respond to precisely coincident spikes (Abeles, 1991; Softky and Koch, 1993; König et al., 1996; Kumar et al., 2010). This view is supported by experimental observations of synchrony in various areas of the nervous system (Usrey and Reid, 1999; Salinas and Sejnowski, 2001). For example, in the retina and the thalamus, neighboring cells are often synchronized at a fine timescale (Alonso et al., 1996; Brivanlou et al., 1998; Usrey et al., 1998; Meister and Berry, 1999); in olfaction, fine odor discrimination relies on transient synchronization between specific neurons (Stopfer et al., 1997).

However, the presence of synchrony does not by itself contradict the view that neural computation can be mainly described in terms of firing rates: it could be that synchrony is incidental and has no significant impact on neural function, if neurons are not very sensitive to it. Therefore, a crucial question to answer is whether neurons are sensitive to coincidences in their inputs, and to what

Received May 18, 2011; revised Sept. 2, 2011; accepted Sept. 6, 2011.

Author contributions: C.R. and R.B. designed research; C.R., S.L., and A.K.M. performed research; C.R. analyzed data; R.B. wrote the paper.

This work was supported by the European Research Council (ERC StG 240132) and by the Swedish Research Council (Grant 80326601)

Correspondence should be addressed to Romain Brette, Equipe Audition, DEC, Ecole Normale Supérieure, 29, rue d'Ulm, 75005 Paris, France. E-mail: romain.brette@ens.fr.

DOI:10.1523/JNEUROSCI.2482-11.2011

Copyright $\odot 2011$ the authors $\quad 0270-6474 / 11 / 3117193-14 \$ 15.00 / 0$ extent. Independently of whether synchrony is functionally useful, this sensitivity determines whether the input-output function of neurons can be essentially described in terms of firing rates.

In a resting neuron, coincidence detection is a trivial property due to the all-or-none nature of neural firing: two coincident postsynaptic potentials (PSPs) superimpose and may reach spike threshold, when two temporally distant PSPs would remain subthreshold. In vivo, when the neuron is subjected to highly fluctuating synaptic activity, coincidence detection is more difficult to quantify. In vivo recordings in neurons of the visual cortex (Usrey et al., 2000) and somatosensory cortex (Roy and Alloway, 2001) with simultaneous recording of a pair of presynaptic neurons have shown that cortical neurons are more likely to fire when the two presynaptic neurons fire together rather than at distant times. However, it could be argued that coincidence between input spikes may not be the cause of increased firing but only correlates with it, since cortical neurons also received inputs from many other cells. In addition, it is not straightforward to understand the implications of these observations when thousands of synaptic inputs are considered.

Previous theoretical studies examined the role of input correlations in neuron models, but with specific input scenarios (Salinas and Sejnowski, 2000; Moreno et al., 2002; Rudolph and Destexhe, 2003b). Here we use a general probabilistic approach to understand and quantify coincidence sensitivity, which allows us to predict the impact of input coincidences on the output firing rate of a neuron, as a function of the statistics of background activity.

\section{Materials and Methods}

\section{Slice preparation and solutions}

Mice of either sex of the CBA $(n=3)$ or the C57BL/6 $(n=5)$ strain aged postnatal day 9-15 were decapitated under sodium-pentobarbital anesthesia in conformity with the rules set by the European Commission Council Directive (86/89/ECC) and approved by the local Swedish Animal Care and Use Committee (Permit N13/10). Transverse slices (250 $300 \mu \mathrm{m}$ ) were collected when the corpus callosum (CC) joins the two hemispheres and the hippocampus covers the underlying subcortical structures (approximately at bregma $-2.5 \mathrm{~mm}$ ) using a vibratome 
(VT1200; Leica). Slices were incubated at $32^{\circ} \mathrm{C}$ in normal aCSF (see below) for 20-30 min, after which they were allowed to cool to room temperature. The cell-dense layer $2 / 3$ region $100-300 \mu \mathrm{m}$ below the pia was targeted in the primary auditory cortex, located $\sim 1.5 \mathrm{~mm}$ dorsal to the rhinal fissure (Fig. 1). Recordings were obtained within $4-5 \mathrm{~h}$ of the preparation.

The low-sodium, high-sucrose aCSF contained the following (in $\mathrm{mM}$ ): $85 \mathrm{NaCl}, 2.5 \mathrm{KCl}$, $1.25 \mathrm{NaH}_{2} \mathrm{PO}_{4}, 25 \mathrm{NaHCO}_{3}, 75$ sucrose, 25 glucose, $0.5 \mathrm{CaCl}_{2}, 4 \mathrm{MgCl}_{2}$, whereas the normal aCSF contained (in $\mathrm{mm}$ ): $125 \mathrm{NaCl}, 2.5$ $\mathrm{KCl}, 1.25 \mathrm{NaH}_{2} \mathrm{PO}_{4}, 26 \mathrm{NaHCO} 3,25$ glucose, 2 $\mathrm{CaCl}_{2}$, and $1 \mathrm{MgCl}_{2}$. These solutions were bubbled continuously with carbogen gas (95\% $\mathrm{O}_{2}-5 \% \mathrm{CO}_{2}$ ), generating a $\mathrm{pH}$ of 7.4 .

The internal pipette solution contained the following (in mM): $130 \mathrm{~K}$-gluconate, $5 \mathrm{KCl}, 10$ HEPES, 1 EGTA, $2 \mathrm{Na}_{2}$-ATP, 2 Mg-ATP, 0.3 $\mathrm{Na}_{3}$-GTP, $10 \mathrm{Na}_{2}$-phosphocreatinine, adjusted to $\mathrm{pH} 7.3$ with $\mathrm{KOH}$, and the osmolarity was $\sim 290 \mathrm{mOsm}$. The recording solution also contained Neurobiotin $(0.1 \%)$ to be able to reconstruct the recording site and the neuron morphology using a standard immunohistochemistry protocol with Streptavidin-Texas Red (Vector Labs).

Recording procedures and data acquisition Slices were transferred to a recording chamber perfused $\left(\sim 3 \mathrm{ml} \mathrm{min}^{-1}\right)$ with oxygenated aCSF at room temperature $\left(25 \pm 2^{\circ} \mathrm{C}\right)$. The putative $\mathrm{Al}$ pyramidal cells were viewed with an upright microscope (Zeiss Axioscope) using a $40 \times$ water-immersion objective (Achroplan, Zeiss) and infrared-differential interference optics equipped with a digital CCD camera (Orca 2, Hamamatsu). Whole-cell current-clamp recordings were done with a Multiclamp 700B amplifier (Molecular Devices) using borosilicate glass microelectrodes with a final tip resistance of $R_{\mathrm{p}}=5-10 \mathrm{M} \Omega$. The signals were filtered with a low-pass 4-pole Bessel filter at $10 \mathrm{kHz}$, sampled at 20 $\mathrm{kHz}$ and digitized using a Digidata 1422A interface (Molecular Devices). Capacitance neutralization was not fully applied, as it was not necessary for this study. The resting membrane potential of recorded cells varied between $-75 \mathrm{mV}$ and $-63.5 \mathrm{mV}$ (average $-70 \mathrm{mV}$ ).

\section{Electrode compensation}

Because we injected highly fluctuating currents (Fig. 2a), the electrode produced artifacts that could not be well corrected with standard bridge compensation (Brette et al., 2008) (Fig. 2b): indeed, in addition to simulated synaptic noise, we injected large exponentially decaying EPSCs (instantaneous rise), and therefore currents were discontinuous. In addition, recordings in the same cell were long (up to a few hours) and electrode properties changed during the course of the experiment (typically, the series resistance increased; cell properties, on the other hand, were stable): in the experiments on coincidence sensitivity in cortical neurons (see Fig. 9b) (done after a couple of hours of recording in the same cell), the series resistance was 70-297 M $\Omega$ (median $120 \mathrm{M} \Omega$ - see Table 1). Thus, even though membrane resistance is high in these cells (several hundred $\mathrm{M} \Omega$ ), it was necessary to subtract the electrode response. Therefore, we used an offline electrode compensation procedure based on an electrode model. Traces are divided in $1 \mathrm{~s}$ slices, and we use a generic model fitting toolbox (Rossant et al., 2011) to fit a linear model of the neuron and electrode to the raw recorded trace:

$$
\begin{gathered}
V_{\text {model }}=V_{\mathrm{n}}+V_{\mathrm{e}} \\
\tau_{m} \frac{d V_{n}(t)}{d t}=V_{r}-V_{n}(t)+R I(t)
\end{gathered}
$$
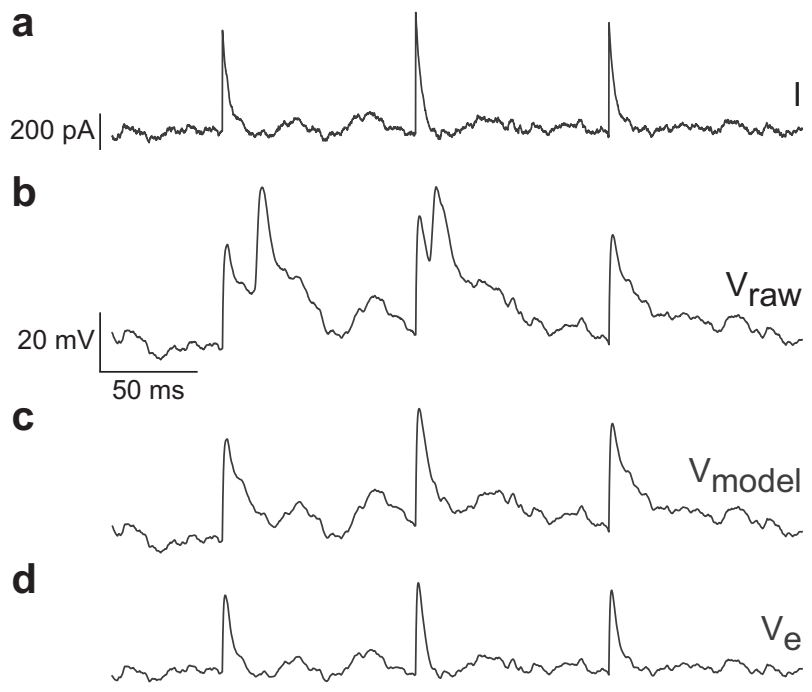

e

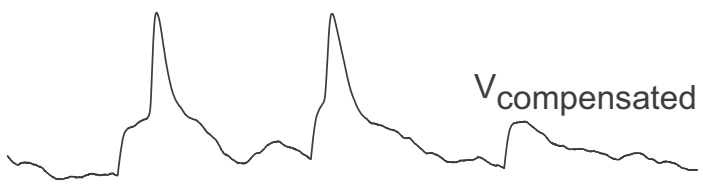

Figure 2. Electrode compensation. $\boldsymbol{a}$, A fluctuating current is injected (here, background noise plus large PS(S). $\boldsymbol{b}$, Raw recorded voltage trace. $\boldsymbol{c}$, A linear model of the neuron and electrode is fitted to the raw trace. Spikes are detected on the difference between raw recording (b) and linear prediction (c).d, Electrode response with the fitted model. $\boldsymbol{e}$, Compensated trace, obtained by subtracting the electrode trace $(\boldsymbol{d})$ from the raw trace $(\boldsymbol{a})$.

$$
\tau_{e} \frac{d V_{e}(t)}{d t}=-V_{e}(t)+R_{s} I(t)
$$

where $\tau_{m}$ and $\tau_{e}$ are the membrane and electrode time constants, $R$ and $R_{s}$ are the membrane and series resistance, and $V_{r}$ is resting potential. These 
Table 1. Distance between mean membrane potential $\left(V_{m}\right)$ and spike threshold, SD of $V_{m}$, and maximum tested PSP in Fig. 9, where a filtered noisy current is injected in the cells (mean \pm SD in the second column)

\begin{tabular}{|c|c|c|c|c|c|c|}
\hline Cell & Current statistics & Threshold - mean $V_{m}$ & SD & Max PSP & Series resistance $R_{s}$ (fit) & Input resistance $R_{i}($ fit) \\
\hline C1 & $50 \pm 30 \mathrm{pA}$ & $17 \mathrm{mV}$ & $5.7 \mathrm{mV}$ & $23 \mathrm{mV}$ & $70 \mathrm{M} \Omega$ & $307 \mathrm{M} \Omega$ \\
\hline $\mathrm{C} 2$ & $10 \pm 30 \mathrm{pA}$ & $21 \mathrm{mV}$ & $9.0 \mathrm{mV}$ & $30 \mathrm{mV}$ & $125 \mathrm{M} \Omega$ & $539 \mathrm{M} \Omega$ \\
\hline $\mathrm{C} 2$ & $10 \pm 10 \mathrm{pA}$ & $25 \mathrm{mV}$ & $3.1 \mathrm{mV}$ & $30 \mathrm{mV}$ & $126 \mathrm{M} \Omega$ & $539 \mathrm{M} \Omega$ \\
\hline C3 & $10 \pm 30 \mathrm{pA}$ & $25 \mathrm{mV}$ & $7.8 \mathrm{mV}$ & $27 \mathrm{mV}$ & $297 \mathrm{M} \Omega$ & $568 \mathrm{M} \Omega$ \\
\hline C4 & $10 \pm 30 \mathrm{pA}$ & $25 \mathrm{mV}$ & $5.6 \mathrm{mV}$ & $20 \mathrm{mV}$ & $85 \mathrm{M} \Omega$ & $399 \mathrm{M} \Omega$ \\
\hline C4 & $50 \pm 30 \mathrm{pA}$ & $16 \mathrm{mV}$ & $5.5 \mathrm{mV}$ & $20 \mathrm{mV}$ & $114 \mathrm{M} \Omega$ & $197 \mathrm{M} \Omega$ \\
\hline C5 & $50 \pm 30 \mathrm{pA}$ & $18 \mathrm{mV}$ & $4.0 \mathrm{mV}$ & $19 \mathrm{mV}$ & $213 \mathrm{M} \Omega$ & $353 \mathrm{M} \Omega$ \\
\hline$C 5$ & $90 \pm 30 \mathrm{pA}$ & $13 \mathrm{mV}$ & $4.9 \mathrm{mV}$ & $19 \mathrm{mV}$ & $219 M \Omega$ & $255 \mathrm{M} \Omega$ \\
\hline C6 & $70 \pm 30 \mathrm{pA}$ & $16 \mathrm{mV}$ & $1.5 \mathrm{mV}$ & $27 \mathrm{mV}$ & $78 \mathrm{M} \Omega$ & $104 \mathrm{M} \Omega$ \\
\hline C6 & $90 \pm 30 \mathrm{pA}$ & $13 \mathrm{mV}$ & $2.3 \mathrm{mV}$ & $27 \mathrm{mV}$ & $124 \mathrm{M} \Omega$ & $93 \mathrm{M} \Omega$ \\
\hline
\end{tabular}

The values in column 2 are used for the theoretical predictions. The series resistance, $R_{s}$, and the cell's input resistance, $R_{i}$ (last column) were obtained with our model fitting technique, applied independently to each recording (see Materials and Methods).
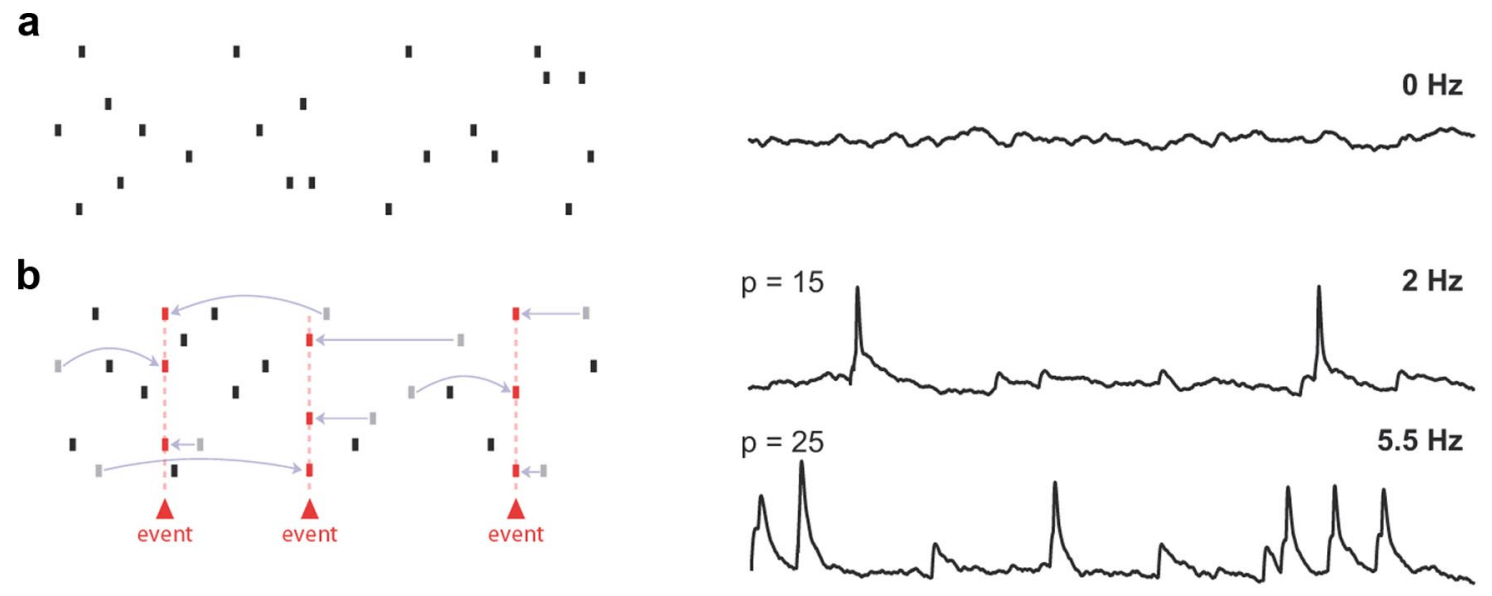

C

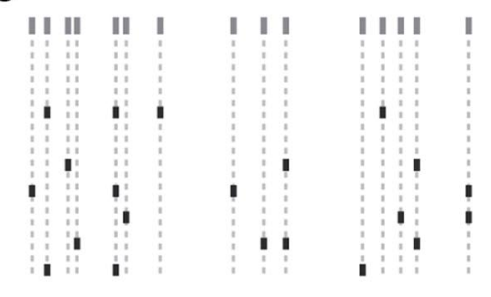

$c=0.002 \quad 3 \mathrm{~Hz}$

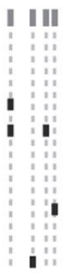

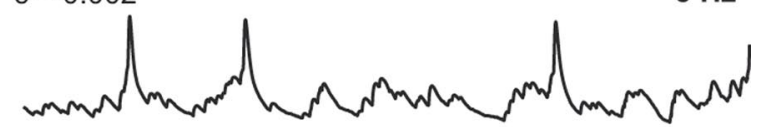

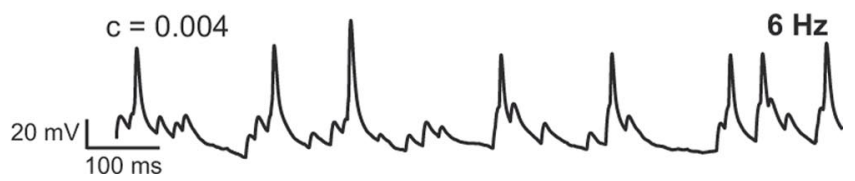

Figure 3. Impact of fine input correlations on the firing rate of a cortical neuron. $a$, Synthetic synaptic currents from 5000 random excitatory $\left(N_{e}=4000\right)$ and inhibitory $\left(N_{i}=1000\right)$ inputs are injected into the cell: the membrane potential is irregular but the cell does not fire. $\boldsymbol{b}$, Inserting random synchrony events (at rate $\lambda_{c}=10 \mathrm{~Hz}$ ) during which $p$ randomly selected synapses are coactivated increases the neuron's firing rate, even though input rates are unchanged and the proportion of synchronous synapses is very low ( 0.4 and $0.6 \%)$. c, Injecting correlated spike trains with homogeneous pairwise correlation c produces similar results: the output firing rate rising very steeply with input correlation. Spike trains were generated by thinning a common reference spike train (Kuhn et al., 2003; Brette, 2009).

parameters are adjusted to minimize the $\mathrm{L}^{\mathrm{p}}$ error between the model prediction $V_{\text {model }}$ (Fig. $2 c$ ) and the raw trace $V_{\text {raw }}$, defined as:

$$
e_{p}=\left(\int\left|V_{\text {model }}(t)-\mathrm{V}_{\text {raw }}(t)\right|^{p}\right)^{1 / p}
$$

with $p<2$. Using an $\mathrm{L}^{\mathrm{p}}$ error rather than the more standard quadratic error reduces the impact of outliers, such as spikes. We detect spikes on the fully compensated trace, $V_{\text {raw }}-V_{\text {model }}$, which corresponds to what is not predicted by the linear model, in particular spikes (Fig. $2 d$ ). We use a manually selected threshold criterion (typically $\sim 20 \mathrm{mV}$ ) to identify spikes. The compensated membrane potential of the cell, which we use for subthreshold analyses, is $V_{\text {raw }}-V_{\mathrm{e}}$ (Fig. 2e).

The electrode compensation technique subtracts the voltage drop through the electrode that is produced by the injected current. However, it does not impact the filtering effect of the electrode, due to the non-zero response time (time constant $\tau_{e}$ ). For this reason, spike height (difference between peak and spike threshold) varied systematically with the estimated electrode time constant $\tau_{e}$ (for cells C1-6: 64, 49, 33, 75, 79, and 57 $\mathrm{mV}$; electrode time constant was $3 \mathrm{~ms}$ for $\mathrm{C} 3$ and $1.1 \mathrm{~ms}$ for $\mathrm{C} 2$, and $<1$ $\mathrm{ms}$ for all other cells; spike duration varied in the opposite direction).

\section{Synaptic currents and data analysis}

Current $A$. To measure the probability that a noisy neuron fires in response to a PSC of varying size $(P(w)$; see Fig. 9), we inject a sum of a background noise and exponentially decaying PSCs. The background noise is an Ornstein-Uhlenbeck process (i.e., low-pass filtered white noise) with time constant $\tau_{\mathrm{N}}=10 \mathrm{~ms}$ (different mean and SD values were tested). After $9 \mathrm{~s}$, we start injecting PSCs every $100 \mathrm{~ms}$, with random size: $\operatorname{PSC}(t)=\alpha w e^{-t / \tau_{s}}$, where $\tau_{\mathrm{s}}=3 \mathrm{~ms}, \alpha=665 \mathrm{pA}$ is a scaling factor (so that maximum depolarization is $\sim 25 \mathrm{mV}$ ), and $w$ is a random number between 0.04 and 1 . The total stimulation lasts $\sim 10 \mathrm{~min}$. 
To compute $P(w)$, we divide the range of values for $w(0.04-1)$ into 20 subintervals, and in each subinterval $i$, we calculate the proportion $\mathrm{p}_{\mathrm{i}}$ of trials in which the neuron spiked within $30 \mathrm{~ms}$ of the injected PSC. The error bar is the SD $\sigma_{i}$ of this estimator: $\sigma_{i}^{2}=p_{i}\left(1-p_{i}\right) /$ $n_{i}$, where $n_{i}$ is the number of data points. Since we are in fact interested in the extra firing probability due to the injected PSC, we subtract the spontaneous firing probability $p_{i}{ }^{0}$, which is estimated from the same trace, but using spikes produced in the $70 \mathrm{~ms}$ preceding each PSC. To compare with theoretical predictions, we measure the spike threshold, the membrane potential distribution and the size of PSPs. The spike threshold is estimated on each trace as the voltage at the maximum of the second derivative of the voltage trace before spikes (Henze and Buzsáki, 2001), and we use the median of all threshold values. The mean and SD of the membrane potential are measured in the first $9 \mathrm{~s}$ of the stimulation (which contains no PSC), after removing spikes, and we assume a Gaussian distribution. To measure the relationship between PSC size and PSP size, we injected a series of 10 identical EPSCs with peak $\alpha=665$ $\mathrm{pA}$, and measured the average peak of PSPs.

Current $B$. To generate synaptic inputs with synchrony events (see Fig. 10), we first generate a set of $N_{e}=4000$ (resp. $N_{i}=1000$ ) independent excitatory (resp. inhibitory) spike trains with Poisson statistics and rate $\lambda_{e}=0.65 \mathrm{~Hz}$ (resp. $\lambda_{i}=1.3 \mathrm{~Hz}$ ). Each spike triggers an exponentially decaying PSC with time constant $\tau_{e}=3 \mathrm{~ms}$ (resp. $\tau_{i}=10 \mathrm{~ms}$ ) and peak value $w_{e}=13 \mathrm{pA}$ (resp. $\left.w_{i}=5.7 \mathrm{pA}\right)$. These values were chosen so that EPSPs and IPSPs are $\sim \pm 1 \mathrm{mV}$ high (close to the average excitatory PSP size in the mouse auditory cortex in vitro; Oswald and Reyes, 2008). Synchrony events are generated according to a Poisson process with rate $\lambda_{c}$, and for each event we pick $p$ excitatory synapses at random and make them simultaneously fire. To compensate, a random spike is suppressed for each new spike. For each cell, we test 4 values of $p(5,15,25,35)$ and 3 values of $\lambda_{c}(5 \mathrm{~Hz}, 10 \mathrm{~Hz}, 20 \mathrm{~Hz})$. The total stimulation lasts $\sim 14 \mathrm{~min}$. We calculate the firing rate $r$ for each pair of values $\left(p, \lambda_{c}\right)$, and the error bar corresponds to the SD assuming Poisson statistics, that is, $r / T$, where $T$ is the duration of the block.

Current $C$. We also measure the firing rate of neurons in response to spike trains with specified pairwise correlation $c$ (Fig. $3 c$; see Fig. 13). The current is the same as in the previous paragraph, except that correlations are introduced differently (Kuhn et al., 2003; Brette, 2009): we generate a single reference Poisson spike train with rate $\lambda / c$, and for each target spike train, we copy every spike of the reference spike train with probability $c$. Correlations are only introduced in excitatory inputs. The stimulation lasts $\sim 5 \mathrm{~min}$, during which we successively test 11 values of $c$ regularly spaced between 0 and 0.01 . The blocks are randomized, so that $c$ does not increase monotonously during the stimulation.

\section{Neuron models}

Models were simulated with the Brian simulator (Goodman and Brette, 2009). We used integrate-and-fire models, where the membrane potential $V(t)$ is governed by the following equation:

$$
\tau_{m} \frac{d V(t)}{d t}=E_{l}-V(t)+I(t)
$$

where $\tau_{\mathrm{m}}=5 \mathrm{~ms}$ is the membrane time constant (taking into account the increase conductance in vivo; Destexhe et al., 2003), $E_{l}=-65 \mathrm{mV}$ is the resting potential and $I(t)$ is the input (current times membrane resistance). The neuron fires when $V(t)$ reaches the threshold $\theta=-55 \mathrm{mV}$, and is then reset to $E_{l}$ and clamped at this value for a refractory period of $5 \mathrm{~ms}$. Despite their simplicity, such simple models can predict the spiking responses of cortical neurons to time-varying currents injected at the
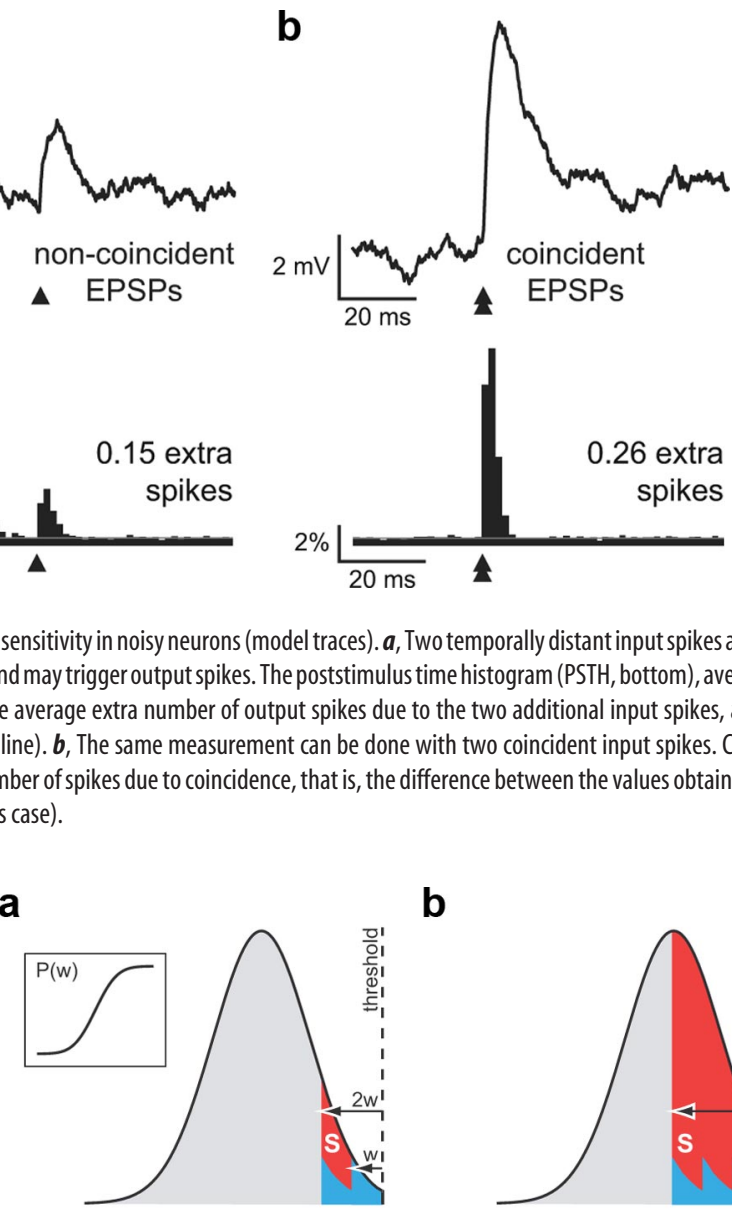

b
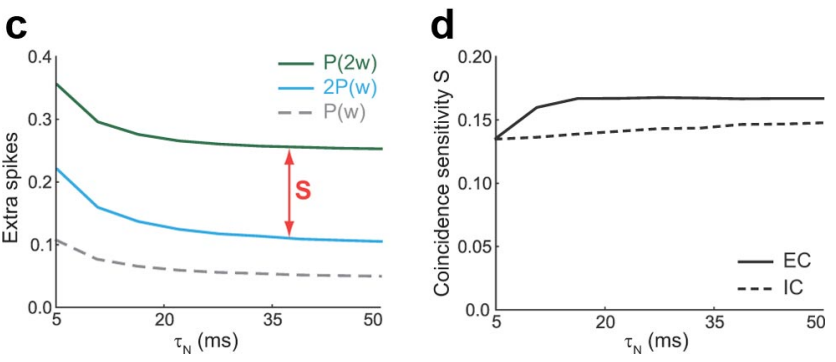

Figure 5. Coincidence sensitivity in a simple probabilistic model. $\boldsymbol{a}$, When excitation and inhibition are balanced, the membrane potential distribution peaks well below the threshold $\theta$ (dashed line). If the neuron is depolarized by an amount $w$, it may fire an extra spike: the expected number of extra spikes is the integral of this distribution between $\theta-w$ and $\theta$, denoted $P(w)$ (inset). The extra number of spikes for two noncoincident spikes is just twice this value, $2 P(w)$ (blue area). The extra number of spikes for two coincident spikes is the integral of the distribution between $\theta-2 w$ and $\theta, P(2 w)$. Coincidence sensitivity is the difference between these two areas: $S=P(2 w)-2 P(w)$ (red). $\boldsymbol{b}$, The same reasoning extends to the analysis of the extra number of output spikes produced by $p$ coincident spikes versus $p$ noncoincident spikes $\left(S_{\mathrm{p}}\right) . c$, In a neuron model with background noise $\left(\sigma_{\mathrm{N}}=4 \mathrm{mV}\right.$ here), this analysis underestimates the true extra number of spikes $P(w)$ due to a depolarization of size $w$ when noise fluctuations are fast ( $\tau_{\mathrm{N}}$ is the time constant of background fluctuations; $w=3.8 \mathrm{mV}$ here). $\boldsymbol{d}$, However, this error has the same magnitude for coincident spikes $(P(2 w))$ and noncoincident spikes $(2 P(w))$, so that the difference $S$ is reasonably accurate. This simple probabilistic model predicts the coincidence sensitivity for PSPs of arbitrary shape, e.g., exponential (with instantaneous currents, IC) or biexponential (with exponential currents, EC), where wis defined as the maximum of the PSP.

soma with surprising accuracy (Gerstner and Naud, 2009; Rossant et al., 2011).

When calculating $P(w)$ and coincidence sensitivity $S$ (Figs. 4-7), a voltage noise is added as an Ornstein-Uhlenbeck process with SD $\sigma$ and 


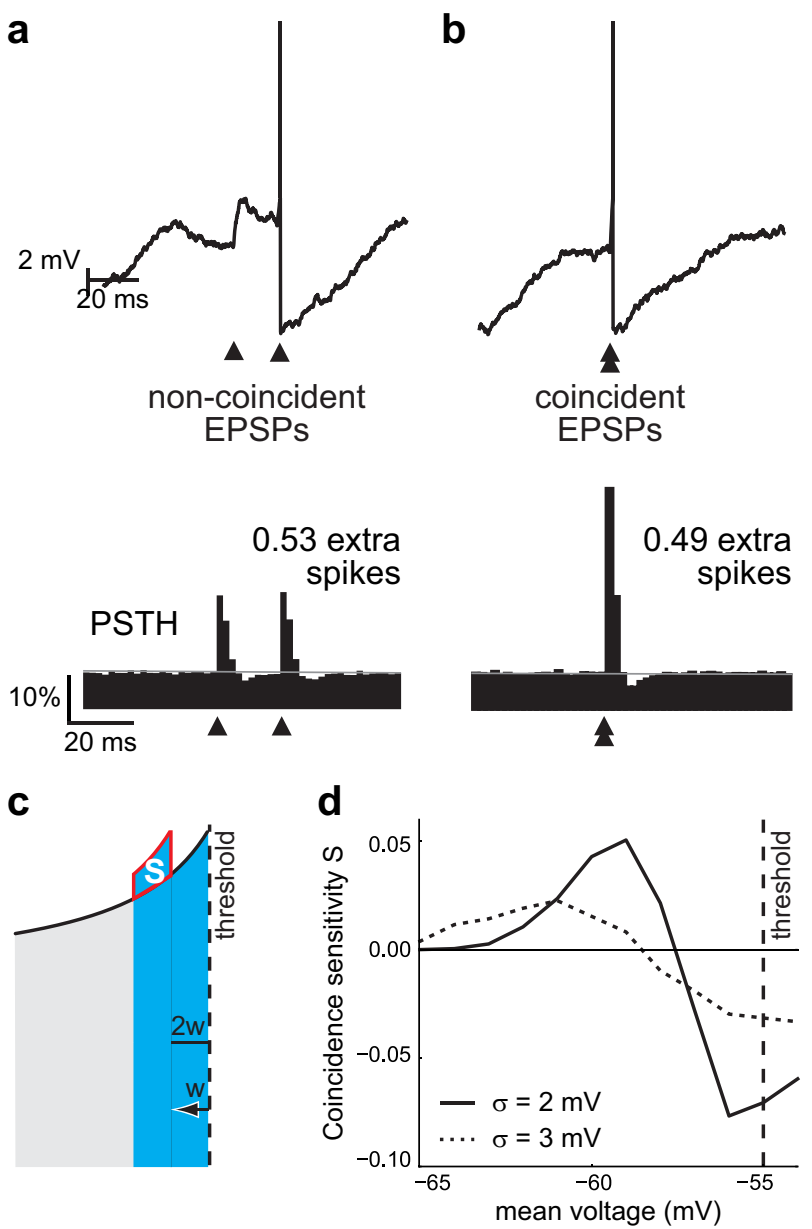

Figure 6. Coincidence sensitivity in the mean-driven versus fluctuation-driven regime. $\boldsymbol{a}, \boldsymbol{b}$, As in Figure 4, we calculate the average extra number of output spikes due to two additional synchronous $(\boldsymbol{b})$ or nonsynchronous $(\boldsymbol{a})$ spikes. The definition is identical but here the neuron is in a mean-driven regime, that is, the average drive exceeds threshold. $c$, In this regime, the membrane potential distribution is increasing near threshold. As a result, coincidence sensitivity $S$ (red contour) is negative, meaning the neuron fires less to coincident than to noncoincident spikes. $\boldsymbol{d}$, Coincidence sensitivity in a neuron model with background noise (with two different SDs, $\sigma$ and $w=1 \mathrm{mV}$ ) as a function of the mean membrane potential, measured in the absence of spikes. The neuron stops being sensitive to coincidences when the mean membrane potential approaches threshold.

time constant $\tau_{\mathrm{N}}$. We estimate $P(w)$, we calculate the probability that the model fires within $10 \mathrm{~ms}$ of an injected PSC, minus the probability that it fires spontaneously in the temporal window. PSCs are either instantaneous (dirac functions), giving exponential PSPs (time constant $\tau_{\mathrm{m}}$ ) or exponential (with time constant $\tau_{e}=3 \mathrm{~ms}$ for excitatory synapses and $\tau_{i}=10 \mathrm{~ms}$ for excitatory synapses), giving biexponential PSPs. The synaptic weight $w$ corresponds to the peak value of the PSPs.

In the simulations with correlated inputs (see Figs. 10, 13, 14), we calculate the inhibitory weight to ensure that the mean total current is zero, which is given by the balance equation:

$$
N_{e} \lambda_{e} \int \operatorname{EPSP}(t)+N_{i} \lambda_{i} \int \operatorname{IPSP}(t)=0
$$

where $\lambda_{e}$ and $\lambda_{i}$ are the excitatory and inhibitory rates. These PSP integrals can be analytically calculated.

In simulations with synaptic conductances instead of currents (see Fig. 12), the input current is:

$$
I(t)=g_{e}(t)\left(E_{e}-V\right)+g_{i}(t)\left(E_{i}-V\right)
$$

where $E_{e}=0 \mathrm{mV}$ and $E_{i}=-75 \mathrm{mV}$ are the excitatory and inhibitory reversal potentials, and $g_{e}(t)$ and $g_{i}(t)$ are the excitatory and inhibitory conductances (in units of the leak conductance). These are sums of exponentially decaying conductances, with the same time constants as before. The membrane time constant was $\tau_{\mathrm{m}}=20 \mathrm{~ms}$. Background activity consists of $N_{e}=4000$ excitatory and $N_{i}=1000$ inhibitory inputs at rate $\lambda_{e}=\lambda_{i}=1 \mathrm{~Hz}$ (Poisson processes). We set the individual peak conductances so that the mean total excitatory conductance is $\left\langle g_{e}\right\rangle=0.5$ (in units of the leak conductance) and the mean total inhibitory conductance is $\left\langle g_{i}\right\rangle=$ 3.25 (which ensures that the mean membrane potential is $-65 \mathrm{mV}$ ).

\section{Theory}

The membrane potential distribution is denoted $p(v)$ and the threshold $\theta$. The probability that the voltage is above $\theta-w$ is:

$$
P(w)=\int_{\theta-w}^{\theta} p(v) d v
$$

The coincidence sensitivity is then $S=P(2 w)-2 P(w)$ for two spikes, and $S_{\mathrm{p}}=P(p w)-p P(w)$ for $p$ spikes. We then assume a Gaussian distribution for $p(v)$ with SD $\sigma$ (this is not a requirement of the theory, but it simplifies calculations), so that:

$$
P(w) \approx \frac{1}{2}\left(1-\operatorname{erf}\left(\frac{\theta-w}{\sigma \sqrt{2}}\right)\right)
$$

where erf is the error function and the spike threshold $\theta$ is relative to the mean membrane potential.

To calculate the extra output rate due to synchrony events in the sparse synchrony scenario (see Fig. 10a), we first calculate the mean and variance of the membrane potential using Campbell's theorems, applied to the nonsynchronous inputs:

$$
\begin{aligned}
\mu & =\left(N_{e} \lambda_{e}-p \lambda_{c}\right) \int \operatorname{EPSP}(t)+N_{i} \lambda_{i} \int \operatorname{IPSP}(t) \\
\sigma^{2} & =\left(N_{e} \lambda_{e}-p \lambda_{c}\right) \int \operatorname{EPSP}^{2}(t)+N_{i} \lambda_{i} \int \operatorname{IPSP}^{2}(t)
\end{aligned}
$$

Then the extra output rate is $\lambda_{c} P(p w)$, where $\mu$ and $\sigma$ are used in the definition of $P$. These formulae are in fact only valid in the subthreshold regime (for a nonspiking neuron), but the change induced by spikes is small when the time constant ( $5 \mathrm{~ms}$ in our simulations) is small compared with the typical interspike interval. More accurate expressions of the membrane potential distribution exist for a limited number of cases (Fourcaud and Brunel, 2002).

When synaptic inputs are modeled as conductances (see Fig. 12), we calculate the membrane potential distribution and EPSP size using the effective time constant approximation (Richardson and Gerstner, 2005), that is, we define the average total conductance as $g_{\text {tot }}=1+\left\langle g_{e}\right\rangle+\left\langle g_{i}\right\rangle$ (in units of the leak conductance) and the effective time constant as $\tau_{\text {eff }}=$ $\tau_{m} / g_{\text {tot }}$, and we use a linear approximation of the membrane equation using these effective parameters:

$$
\tau_{\text {eff }} \frac{d V(t)}{d t}=E_{l}-V(t)+\frac{1}{g_{\text {tot }}}\left(g_{e}(t)\left(E_{e}-V_{0}\right)+g_{i}(t)\left(E_{i}-V_{0}\right)\right)
$$

where $V_{0}$ is the average membrane potential:

$$
V_{0} \approx \frac{E_{L}+\left\langle g_{e}\right\rangle E_{e}+\left\langle g_{i}\right\rangle E_{i}}{1+\left\langle g_{e}\right\rangle+\left\langle g_{i}\right\rangle}
$$

With this approximation, which takes into account the change in effective time constant and resistance, we can use exactly the same analytical methods as before (calculate the EPSPs analytically, and use Campbell's 
theorems to calculate the membrane potential distribution). It is possible to calculate the membrane distribution more precisely with more sophisticated methods (Rudolph and Destexhe, 2003a; Richardson and Gerstner, 2006; Richardson and Swarbrick, 2010), but this simple method was sufficient in our case.

\section{Results}

To motivate this study, Figure 3 shows the sensitivity of a cortical neuron (layer $2 / 3$ of primary auditory cortex, Fig. 1) to fine correlations in its inputs. We injected a fluctuating current in vitro composed of a sum of 4000 excitatory and 1000 inhibitory random spike trains (Fig. $3 a$, each presynaptic spike triggers a postsynaptic current), with Poisson statistics. Excitation and inhibition were balanced on average, as in the sensory cortex in vivo (Destexhe et al., 2003), and this cell did not fire when the inputs were uncorrelated (Fig. 3a). We introduced synchrony events where $p$ randomly selected presynaptic excitatory spikes occurred at the same time (Fig. 3b), without changing individual spike train statistics. The neuron fired at $2 \mathrm{~Hz}$ when synchrony events involved only $p=15$ synapses, that is, $<0.4 \%$ of all synapses, and it fired at 5.5

$\mathrm{Hz}$ with $p=25$. This exquisite sensitivity was also seen when homogeneous pairwise correlations with smaller higher-order correlations were introduced in the inputs (Fig. $3 c$ ): the cell fired at $3 \mathrm{~Hz}$ with input correlation $c=0.002(0.2 \%)$ and $6 \mathrm{~Hz}$ with $c=$ 0.004 . Thus, pairwise correlations that are so small that they could probably not even be observed in a paired recording (a pair of coincident spikes between two given presynaptic neurons occurs every $150 \mathrm{~s}$ with $c=0.004$ and firing rate $F=0.6 \mathrm{~Hz}$ ) still have a dramatic impact on postsynaptic firing rate. We observed the same phenomenon in simple integrate-and-fire neuron models, which suggests that it does not rely on specific cellular mechanisms.

\section{Coincidence sensitivity: a simple probabilistic model}

How general is this property? To quantify coincidence sensitivity, we compare the impact of two coincident versus two noncoincident spikes on a neuron with noisy background synaptic activity (Fig. 4). If two input spikes are added on top of the background activity, the neuron will fire more spikes on average. This average extra number of spikes can be measured by repeating the same protocol over many trials and computing the poststimulus time histogram (PSTH): the extra number of spikes is the integral of the PSTH above the baseline (Fig. $4 a$, yellow line). This corresponds to the "spike efficacy" defined by Usrey et al. (2000). In Figure 4 , the neuron model fired on average 0.15 extra spikes in response to two noncoincident spikes and 0.26 extra spikes in response to two coincident spikes. Therefore, the average extra number of spikes due to input coincidence was $S=0.26-0.15=$ 0.11 spikes. We define this quantity as the coincidence sensitivity, $S: S>0$ means that the neuron fires more when its inputs are coincident. It depends on neuronal and synaptic properties, and on the statistics of background activity.

Coincidence sensitivity can be quantified with a simple probabilistic approach (Fig. 5). When excitation and inhibition are
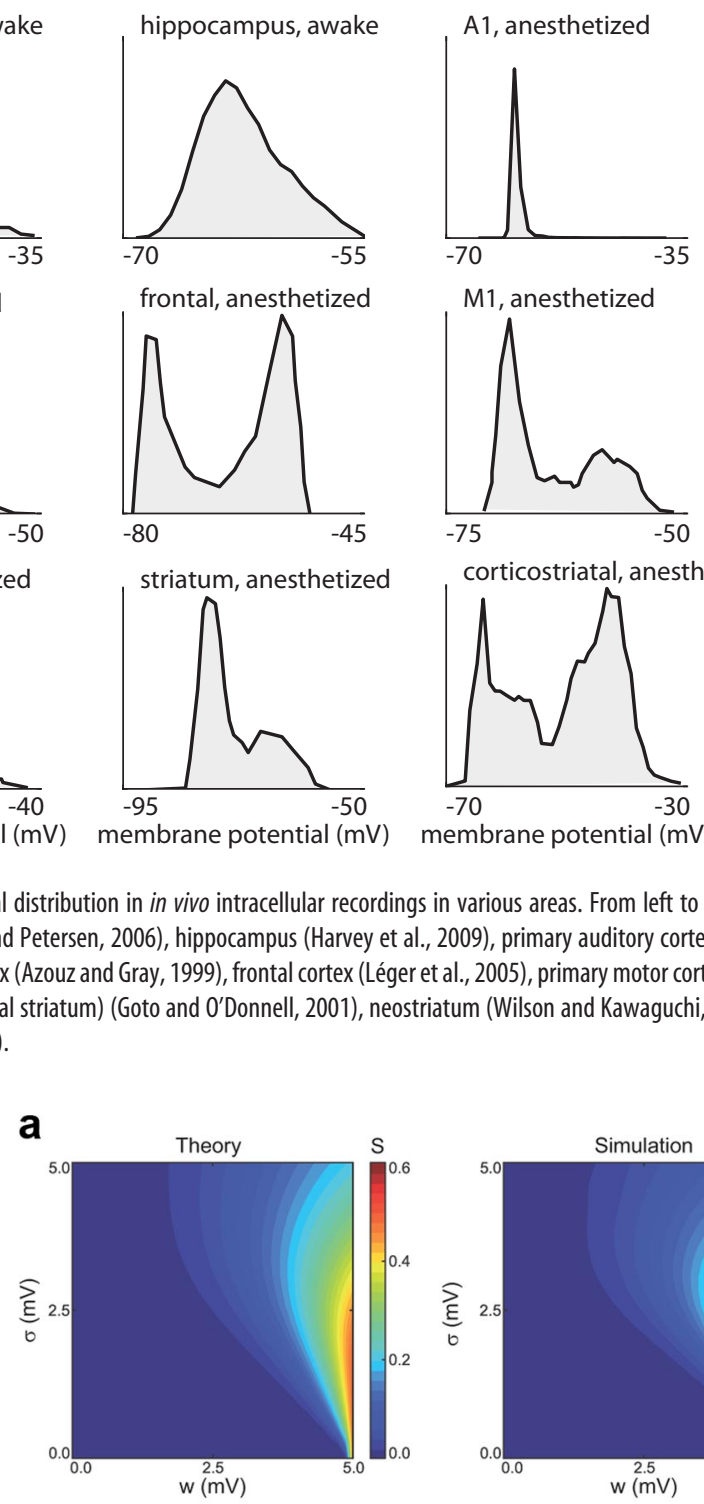

$\mathrm{M1}$, anesthetized

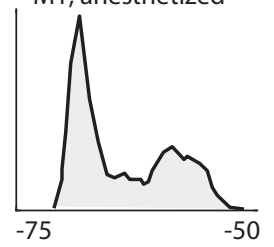

striatum, anesthetized
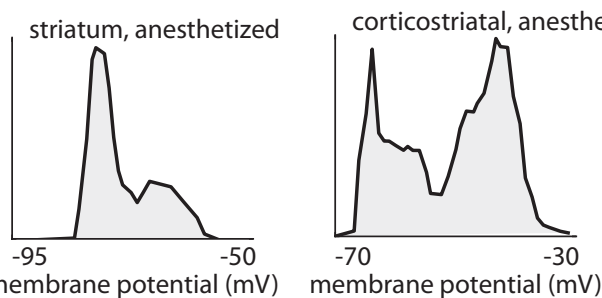

membrane potential $(\mathrm{mV})$
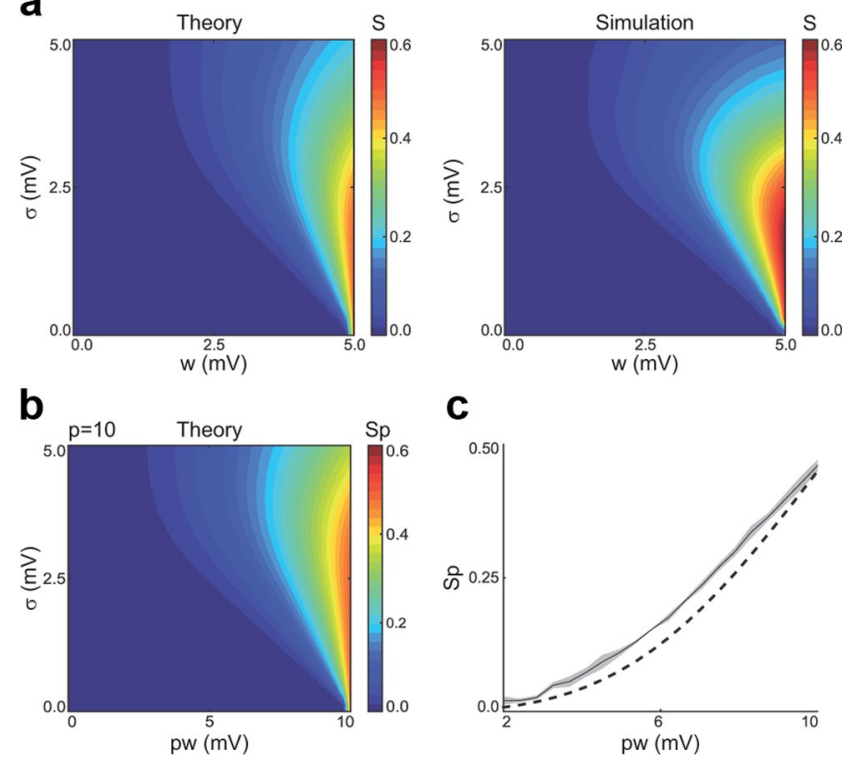

C

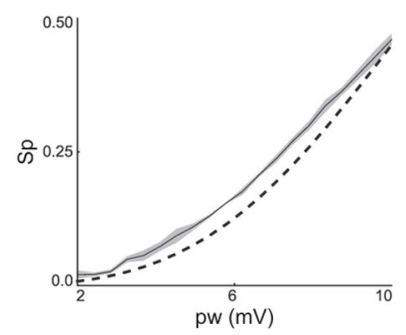

Figure 8. Influence of background noise on coincidence sensitivity in a neuron model. $\boldsymbol{a}$, Coincidence sensitivity (color-coded) as a function of the SD $\sigma$ of the membrane potential and the PSP size $w$, according to our theory (probabilistic model of Fig. 5) and measured in numerical simulations. The threshold was $10 \mathrm{mV}$ above the mean membrane potential and the time constant of background fluctuations was $\tau_{\mathrm{N}}=15 \mathrm{~ms}$. $\boldsymbol{b}$, Coincidence sensitivity $S_{p}$ for $p$ coincident versus noncoincident spikes, as a function of $\sigma$ and the total depolarization pw, for $p=$ 10.c, Coincidence sensitivity $S_{p}$ for $\sigma=4 \mathrm{mV}$ as a function of $\mathrm{pw}$, when both $p$ and $w$ are varied (gray area). The dashed line shows the theoretical prediction for many small PSPs $S_{\infty}$.

balanced on average, the membrane potential distribution peaks well below threshold (Fig. $5 a$ ). The neuron fires in response to an input spike if its membrane potential $V_{\mathrm{m}}$ is close enough to the spike threshold $\theta$. More precisely, if $\mathrm{w}$ is the size of the postsyn- 


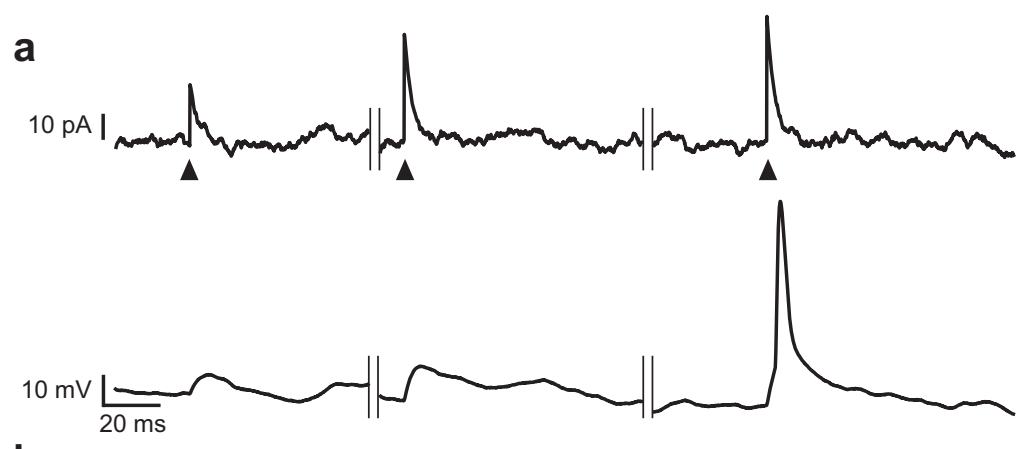

b

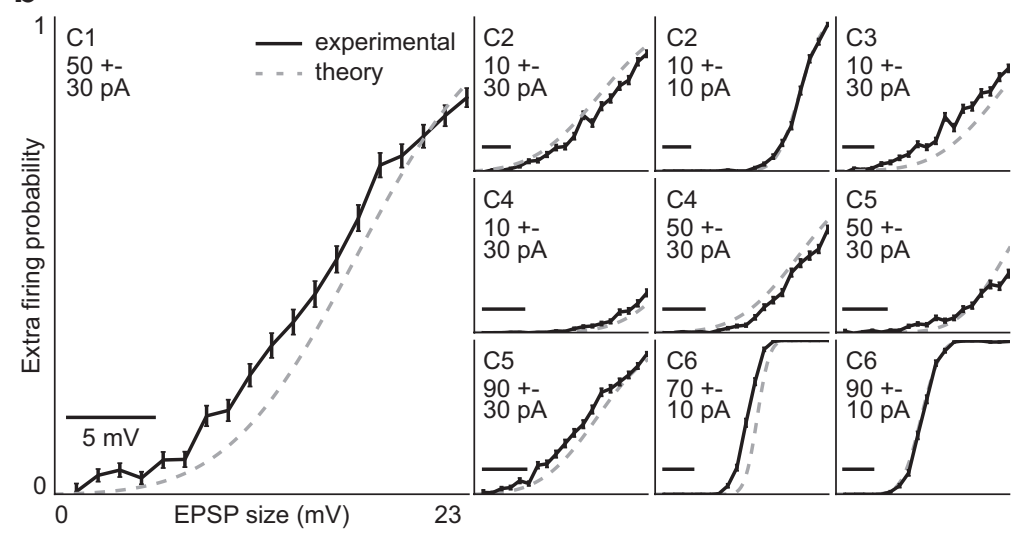

C

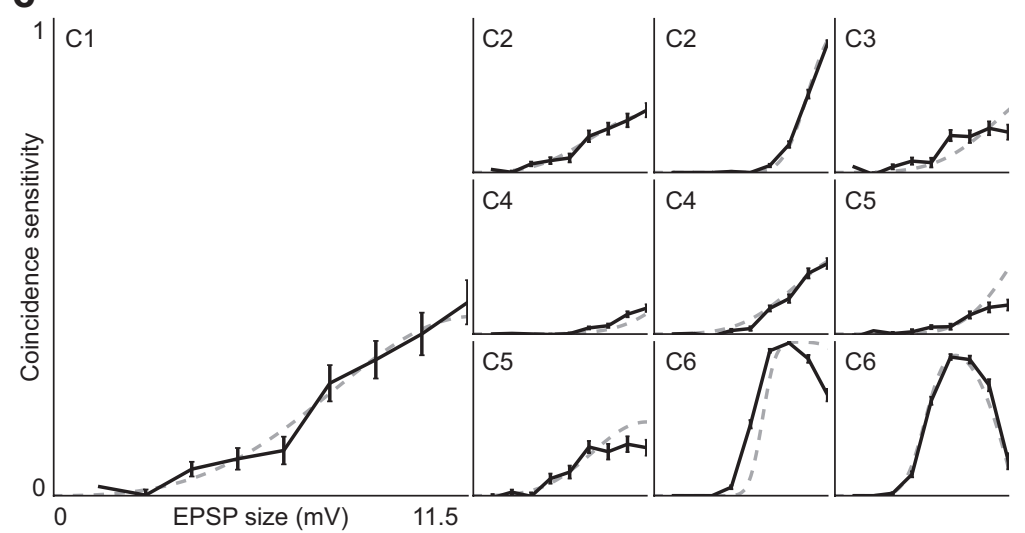

Figure 9. Coincidence sensitivity in cortical neurons. $\boldsymbol{a}$, PSCs of various sizes are injected on top of a background noisy current, and the probability of firing $P(w)$ is measured as a function of measured PSP size $w$. $\boldsymbol{b}$, Firing probability $P(w)$ in 6 cells for different noise statistics (numbers: mean \pm SD of background current). Measurements are compared with theoretical predictions with the probabilistic model, using the measured threshold. Note that the horizontal scale differs between cells because they have different membrane resistances (horizontal bar: $5 \mathrm{mV}$ ). Table 1 indicates the maximum PSP size, spike threshold and membrane potential statistics for each cell. c, Coincidence sensitivity $S=P(2 w)-2 P(w)$, measured and predicted, in the same cells. Note that the horizontal scale is half the scale in $\boldsymbol{b}$.

aptic potential (PSP), then the neuron fires if $V_{\mathrm{m}}+w>\theta$. Graphically, the probability $P(w)$ that the neuron fires (Fig. $5 a$, inset) is then the integral of the membrane potential distribution between $\theta$ and $\theta-w$. If two temporally distant spikes are received, the probability that the neuron fires is just $2 P(w)$, corresponding to the orange area in Figure $5 a$. If two coincident spikes are received, the probability that the neuron fires is $P(2 w)$, that is, the integral of the membrane potential distribution between $\theta$ and $\theta-2 w$. Thus, the coincidence sensitivity is the difference between these two probabilities: $S=P(2 w)-2 P(w)$, corresponding to the red area in Figure $5 a$. This approach extends to the coincidence sensitivity with $p$ spikes $S_{p}$, defined as the difference in the average extra number of spikes with $p$ coincident versus noncoincident input spikes (Fig. 3b).
This simplified description is not entirely accurate, because an input spike may bring the membrane potential slightly below threshold, which would not immediately trigger an output spike but still increase the probability of firing at a later time, because of background fluctuations. Therefore, our description is an approximation that is valid when the duration of a PSP is short compared with the time constant of background fluctuations, and otherwise underestimates the true probability $P(w)$. This is shown in Figure $5 c$, where $P(w)$ was numerically estimated in a neuron model with background noise, as a function of the time constant $\tau_{\mathrm{N}}$ of that noise (dashed line). The theoretical prediction with the probabilistic model corresponds to the asymptotic value for large $\tau_{\mathrm{N}}$. For fast fluctuations ( small $\tau_{\mathrm{N}}$ ), the true value of $P(w)$ is significantly larger than our prediction. However, the error we make has the same magnitude and sign for $P(2 w)$ (two coincident spikes) and for $2 P(w)$ (two noncoincident spikes), so that the coincidence sensitivity $S$, which is the difference, is in fact well approximated by our simple probabilistic model (Fig. 5d).

\section{Fluctuation-driven versus mean-driven regime}

In Figure 5, the neuron responds more to coincident than to noncoincident spikes $(S>0)$ because the membrane potential is more likely to be near average than near threshold, i.e., the membrane potential distribution $p\left(V_{\mathrm{m}}\right)$ is decreasing. This reflects the fact that the neuron was in a "fluctuation-driven" regime, because of the balance between excitation and inhibition. If the average synaptic current is suprathreshold (more excitation than inhibition), then the situation is reversed (Fig. 6). Indeed, in this case the neuron fires regularly at a rate defined by the average current ("mean-driven" regime) and spends more time near threshold than far from it (Fig. $6 a, b$ and membrane potential distribution in Fig. $6 c$, see voltage traces). It follows that two coincident spikes have a smaller impact on output firing than two noncoincident spikes, that is, $S<0$ (Fig. $6 c$, area with red contour). Figure $6 d$ shows the measured coincidence sensitivity of a neuron model with background noise where the mean was varied, for two different noise variances. Coincidence sensitivity is positive when the average membrane potential (measured without threshold) is far below threshold, and it changes sign when it approaches threshold (more precisely, $S$ is expected to change sign when the average membrane potential is within 2 PSPs of the threshold_-PSP size was $1 \mathrm{mV}$ in this figure). Thus, neurons are sensitive to coincidences in the fluctuation-driven (or balanced) regime, not in the mean-driven regime.

Which one of these two situations is a better description of membrane potential dynamics in vivo, fluctuation-driven or 
a

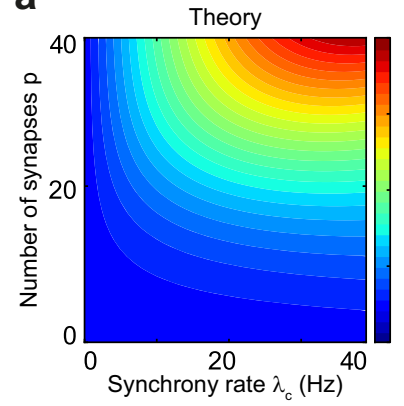

C

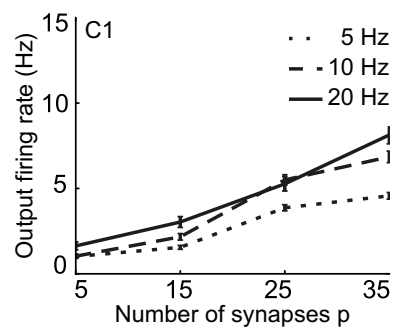

Simulation

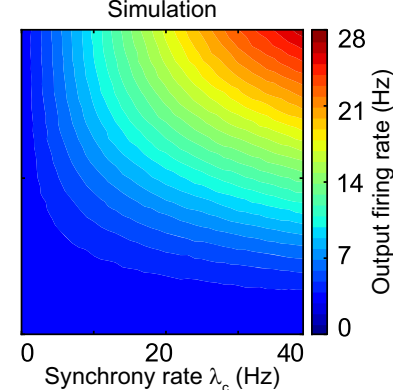

b

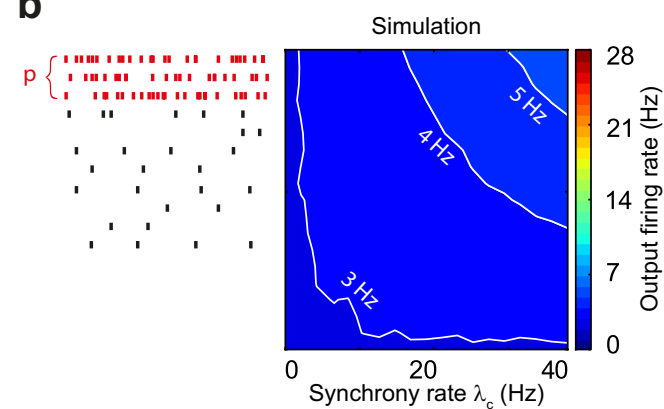

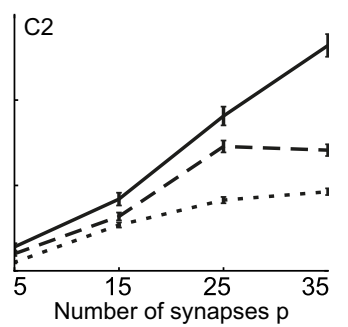
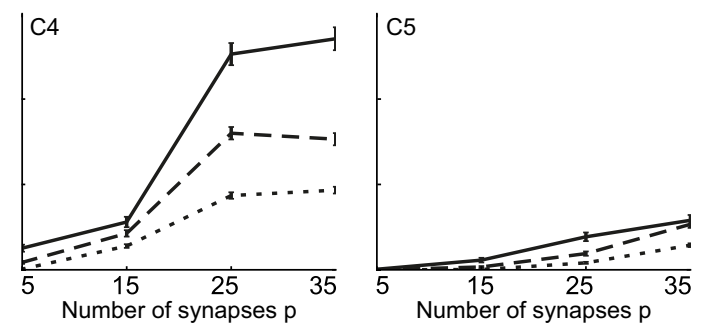

Figure 10. Impact of sparse synchrony on the firing rate of neurons. $\boldsymbol{a}$, Firing rate (color-coded) of a neuron model in response to random input spike trains with rates $1 \mathrm{~Hz}$ with synchrony events, as described in Figure $3 b$. Events occur at rate $\lambda_{c}$ and consists of $p$ synchronous spikes (out of 4000 excitatory synapses). Excitatory and inhibitory PSPs are $0.5 \mathrm{mV}$ and $-2 \mathrm{mV}$ high, respectively (so that the mean current is zero). $\boldsymbol{b}$, Firing rate of the same model in response to random (uncorrelated) spike trains where the input rate of $p$ inputs are changed to $\lambda_{c}$. $\boldsymbol{c}$, Firing rate of 4 cortical cells with the same inputs as in $\boldsymbol{a}$, as a function of number $p$ of synchronous synapses in each event, for 3 different synchrony event rates (PSP size was $0.4-0.6 \mathrm{mV}$ and threshold $15-25 \mathrm{mV}$ above the mean membrane potential). Note that cell 5 had a lower membrane resistance than other cells.

mean-driven? In Figure 7, we show membrane potential distributions in in vivo intracellular recordings in various areas, extracted from a number of previous studies. In anesthetized preparations, these distributions are often bimodal, which reflects "up" and “down" states (Constantinople and Bruno, 2011). Since down states are quiescent, only the depolarized mode (up state) is relevant to this study. In all cases, the membrane potential distribution decreases toward threshold, suggesting that neurons are in a fluctuation-driven rather than mean-driven regime. Therefore, we now focus on the fluctuation-driven regime.

\section{Influence of background noise statistics}

Quantitatively, coincidence sensitivity depends on PSP size and background noise statistics (e.g., mean and variance of the membrane potential). In our probabilistic model, $S$ increases monotonically with PSP size $w$ (Fig. $8 a$, left), until $2 \mathrm{w}$ is the difference between average membrane potential and threshold $(10 \mathrm{mV}$ in Fig. $8 a$ ), which corresponds to the inflection point of the sigmoid $P(w)$ (Fig. 5a, inset). The relationship with the SD $\sigma$ of the membrane potential is more surprising: it appears that $S$ is maximal for an intermediate value of $\sigma$, for example $\sim 2 \mathrm{mV}$ when $w \approx 5$ $\mathrm{mV}$. Intuitively, it can be explained as a trade-off: if there is little background noise ( $s m a l l \sigma$ ), then two spikes are unlikely to make the neuron fire, whether they are coincident or not, and therefore $S$ is small; if there is too much noise (large $\sigma$ ), then the membrane potential distribution is flat (all voltages are equally likely) and two coincident spikes have the same effect as two noncoincident spikes. These theoretical predictions accurately matched numerical results obtained in neuron models with simulated input currents (Fig. $8 a$, right).

It could be argued that $5 \mathrm{mV}$ is unreasonably large for a PSP. However, the same analysis applies when considering $p$ small PSPs instead of 2 large PSPs (Fig. $8 b, p=10$ and $w$ is varied between 0 and $1 \mathrm{mV}$ ): 10 coincident PSPs of $1 \mathrm{mV}$ produce on average 0.5 more output spikes than if they were not coincident. In fact, when the number of input spikes $p$ is large, the coincidence sensitivity $S_{p}$ is mainly determined by the total depolarization $p w$, because small isolated PSPs have little effect on output firing (Fig. $5 b$ : the orange area is small). Figure $8 c$ shows the relationship between $S_{p}$ and $p w$ for different values of $p$ (between 10 and 30) and with $w$ varying between 0 and $10 \mathrm{mV} / p$ (shaded blue), in a simulated neuron model. The dashed line shows the theoretical prediction with $p=\infty$ (i.e., many small PSPs).

\section{Coincidence sensitivity in vitro}

We then verified our theoretical predictions in auditory cortical neurons in vitro (Fig. 9). We generated background noisy currents with specified mean and $\mathrm{SD}$, and injected them into the soma with additional exponentially decaying currents of various sizes, representing EPSCs, PSCs (Fig. 9a) (we address the more realistic case of synaptic conductances below). We recorded the spikes produced by the cell in response to this stimulation and calculated the probability $P(w)$ that the cell fires as a function of PSP size $w$ (Fig. 9b). To compare with our theoretical predictions, we measured the membrane potential distribution in the cell and the spike threshold (see Materials and Methods). Our simple probabilistic model could predict $P(w)$ with good accuracy over the tested range of background statistics (Fig. $9 b$ ). The theoretical prediction tends to slightly underestimate $P(w)$, as we already discussed previously (Fig. 5). From the measured $P(w)$, we could calculate the coincidence sensitivity $S=P(2 w)-2 P(w)$, which was also well predicted by our theory (Fig. $9 c)$. Consistently with our theoretical analysis, all cells $(n=6)$ were very sensitive to coincidences.

\section{Impact of sparse synchrony events on output firing}

To understand the impact of this property when many synaptic inputs are considered, we analyzed the sparse synchrony scenario 
a
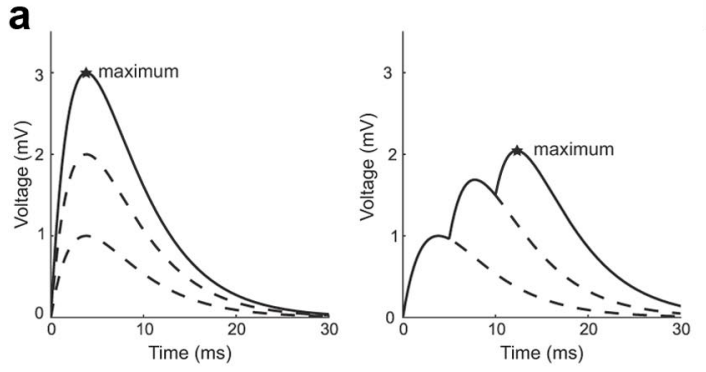

b

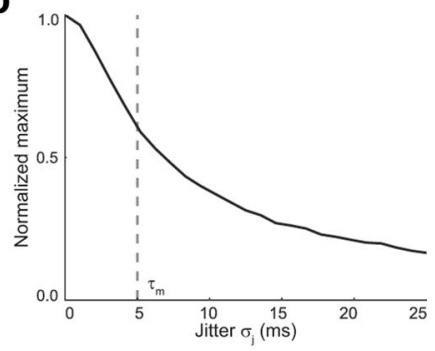

C

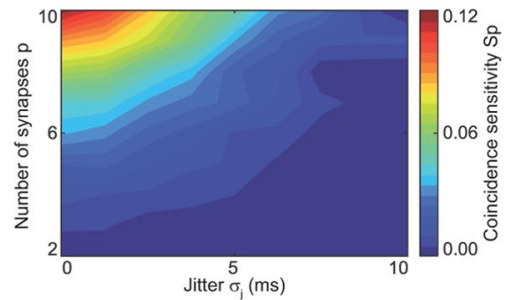

$\alpha=0.5$

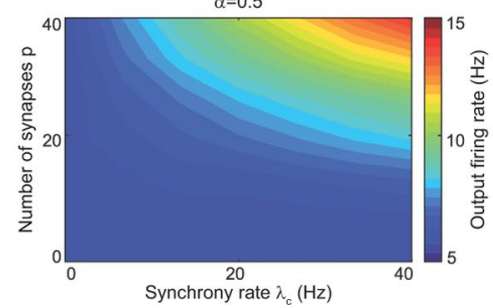

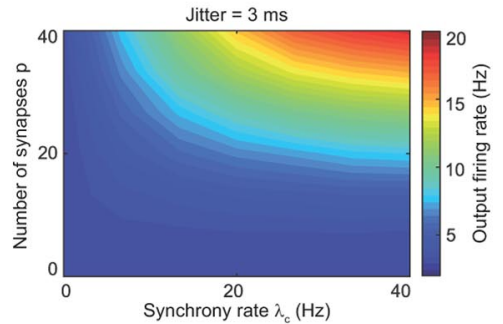

e

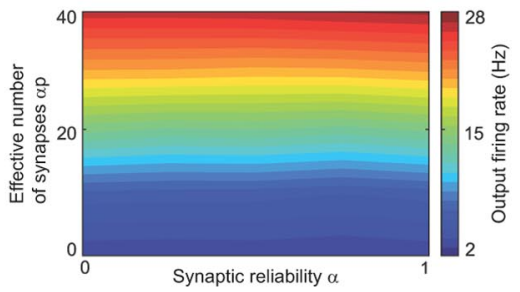

Figure 11. Effect of spike jitter and synaptic failure on coincidence sensitivity. $\boldsymbol{a}$, When random jitter is introduced in input spikes, the sum of coincident PSPs has a smaller maximum, which is determined (on average) by the amount of jitter. $\boldsymbol{b}$, This maximum (numerically calculated for many coincident biexponential PSPs) decreases with the SD of the jitter, reaching $50 \%$ of its maximum value when the jitter is comparable to the membrane time constant. c, As a result, neuron models are sensitive to coincidences when the jitter is smaller than the membrane time constant, not when it is larger (left; $\tau_{\mathrm{N}}=15 \mathrm{~ms}, \sigma_{\mathrm{N}}=4 \mathrm{mV}, w=0.5 \mathrm{mV}$ ). For a jitter of $3 \mathrm{~ms}$ and a membrane time constant $\tau_{\mathrm{m}}=5 \mathrm{~ms}$, the effect of sparse synchrony on output firing rate is qualitatively similar to the effect obtained with zero-lag synchrony ( 0 ms jitter, Fig. 10). $\boldsymbol{d}$, If synapses transmit presynaptic spikes with probability $\alpha=50 \%$ (i.e., failure probability is $50 \%$ ), the effect of sparse synchrony is still qualitatively unchanged. $\boldsymbol{e}$, In this case, the impact on output firing rate is essentially determined by the effective number of synchronous synapses in each event, seen from the postsynaptic side, which is on average $\alpha p$ (vertical axis), and does not depend otherwise on synaptic transmission probability $\alpha$ (horizontal axis).

presented in Figure $3 b$ with our theoretical approach (Fig. 10a). In that scenario, the neuron receives 4000 excitatory and 1000 inhibitory spike trains with Poisson statistics. Excitation and inhibition are balanced (mean total current is zero), so that the neuron fires irregularly at low rate $(3 \mathrm{~Hz})$. Synchrony events are introduced at random times (also with Poisson statistics), by shifting $p$ random excitatory spikes to the event time. This protocol leaves individual spike train statistics unchanged but modifies the correlations. We can use our probabilistic model to predict the extra firing rate due to these synchrony events (see Materials and Methods) (Fig. 10a, left), and the prediction agrees very well with numerical simulations (Fig. 10a, right). The impact of synchrony on output firing rate is dramatic: by introducing synchrony between $<1 \%$ of all synapses without changing the input firing rates, the output firing rate increases from $2 \mathrm{~Hz}$ to 28 $\mathrm{Hz}$ in Figure $10 a$. On the other hand, if we increase the firing rate of $p$ excitatory inputs without changing the correlations while maintaining the excitatory-inhibitory balance (by increasing the inhibitory rate), then the output firing rate hardly changes (Fig. $10 b)$. This is not so surprising since the total rate of excitatory input spikes is hardly modified when the rates of only $1 \%$ of synapses are increased and therefore the variance of the membrane potential changes very little (precisely, the relative change is $1+\left(p \lambda_{c}\right) /\left(N_{e} \lambda_{e}\right)$, where $\lambda_{c}$ is the rate of the $p$ inputs, $\lambda_{e}$ is the initial excitatory rate and $N_{e}$ is the number of excitatory inputs).

We then tested the impact of sparse synchrony in cortical cells, by injecting synthesized currents made of sums of excitatory and inhibitory PSCs with synchrony events (Fig. 10c). In all tested cells $(n=4)$, we observed that inserting synchrony events without changing input rates had a dramatic impact on the cell's firing rate, with only a few synapses involved in each synchrony event $(<1 \%$ in all cases). Firing rates tended to be lower than in model simulations (Fig. 10a) because the spike threshold was high in these cells ( $>30 \mathrm{mV}$ above resting potential), and perhaps because of adaptive properties, which were not included in the models.

\section{Effect of temporal jitter and synaptic unreliability}

In our analysis, we compared precisely coincident spikes with temporally distant spikes. How does coincidence detection depend on the delay between input spikes, that is, on the temporal precision of coincidences? Consider a sum of coincident PSPs, and introduce a temporal jitter in spike times (Fig. 11a): the peak voltage decreases with the amount of jitter $\sigma_{j}$ (defined as the SD of spike times). It is possible to calculate the average peak voltage as a function of $\sigma_{j}$ (Fig. $11 b$ ): it decreases with a characteristic time close to the decay time constant of PSPs, that is, close to the membrane time constant $\tau_{\mathrm{m}}$ (dashed line). In our theoretical model, coincidence sensitivity is determined by the peak size of combined PSPs, therefore we expect neurons to be sensitive to coincidences when the temporal jitter is smaller than $\tau_{\mathrm{m}}$. This is confirmed by numerical simulations (Fig. 11c): coincidence sensitivity $S_{p}$ quickly increases with the number of input spikes when $\sigma_{j}<\tau_{\mathrm{m}}$ (left; $\tau_{\mathrm{m}}=5 \mathrm{~ms}$ here), and introducing a 3 ms temporal jitter does not significantly change the impact of sparsely synchronous inputs on output firing rate (Fig. 11, right).

An additional source of variability in vivo is the probabilistic nature of synaptic transmission: presynaptic spikes are transmitted with some probability $\alpha<1$. However, it does not degrade the temporal precision of coincidences, and therefore this fact does not qualitatively impact coincidence sensitivity (Fig. 11d). For example, when synapses transmit spikes with probability 0.5 , sparse synchrony still has a dramatic impact on output firing rate (left). For a synchrony event consisting of $p$ presynaptic spikes, on average $\alpha p$ synchronous spikes are seen on the postsynaptic side, and therefore the output firing rate is essentially determined by the effective number of synchronous synapses $\alpha p$, with little dependence on transmission probability $\alpha$ (Fig. $11 d$, right). Therefore, the impact of stochastic synaptic transmission on coincidence detection is simply to increase the number of synchronous presynaptic spikes for a given postsynaptic effect by a factor $1 / \alpha$. 


\section{Effect of synaptic conductances}

In all the results we have shown, the synaptic inputs were modeled as currents rather than conductances. Considering the more realistic case of synaptic conductances has two main consequences: 1) the total conductance is increased (by a factor of 5; Destexhe et al., 2003) and therefore EPSP size is decreased by the same amount, 2) the effective membrane time constant is reduced in the same proportion. In our models, we considered a short membrane time constant ( $\sim 5 \mathrm{~ms})$ to take this effect into account. For the problem we are considering, the sensitivity to excitatory coincidences, this is likely to be sufficient. First, the excitatory reversal potential is high compared with the spike threshold (typically $E_{e} \approx 0 \mathrm{mV}$ ), and therefore the driving force is not very variable. Second, our measurements of coincidence sensitivity only involve a few input spikes ( 2 in Fig. $5 a$, a few tens in Fig. $10)$, and therefore these additional spikes should have virtually no effect on the total conductance (always $<1 \%$ ).

To demonstrate this point, we measured the coincidence sensitivity of a neuron model with a background of random excitatory and inhibitory input spikes (Fig. 12a), with the same statistics as in Figure 10 (with no correlations), except the inputs were modeled as synaptic conductances: the excitatory (resp. inhibitory) current is $I_{e}(t)=g_{e}(t)\left(E_{e}-V\right)$ (resp. $I_{\mathrm{i}}(t)=$ $g_{i}(t)\left(E_{i}-V\right)$ ), where $E_{e}=0 \mathrm{mV}$ (resp. $E_{i}=-75 \mathrm{mV}$ ) is the excitatory (resp. inhibitory) reversal potential. The mean excitatory conductance was half the leak conductance, while the mean inhibitory conductance was 3.25 times the leak conductance. Thus, the total conductance was $\sim 5$ times the leak conductance, so that the effective membrane time constant was $\sim 5$ times smaller than the membrane time constant $\left(\tau_{\text {eff }}=\tau_{\mathrm{m}} /(1+0.5+\right.$ $3.25)$ ). We then measured the coincidence sensitivity by injecting excitatory input spikes with variable amplitude, as in Figure 9 (again, the inputs were modeled as conductances) (Fig. 12b). We made theoretical predictions with the same formulae as before, but we used the effective time constant and resistance to (approximately) calculate the membrane distribution and EPSP size. In this effective time constant approximation, the driving forces are replaced by their average $\left(E_{\mathrm{e}}-V_{0}\right.$ and $\left.E_{\mathrm{i}}-V_{0}\right)$ and the leak conductance is replaced by the mean total conductance (Richardson and Gerstner, 2005). We then used exactly the same methods as before. It is possible to calculate the membrane distribution more precisely (Richardson and Gerstner, 2006; Richardson and Swarbrick, 2010), but this simple current-based method was already accurate enough in our case. Figure $12 b$ shows that our theoretical prediction remains reasonably accurate in this complex situation, which is far from the ideal setting (synaptic conductances rather than currents, shot noise rather than diffusion).

\section{Impact of input correlations without synchrony events}

One may argue that the sparse synchrony scenario we described in Figure 10 is rather specific in that we introduce strong depolarizations, which also increases higher order correlations (al- though this is consistent with intracellular recordings in the auditory cortex in vivo; DeWeese and Zador, 2006). However, the results still hold if correlations are introduced in a way that minimizes these higher orders, as in Figure $3 c$ : a fixed pairwise correlation $c$ is introduced between all excitatory spike trains with firing rate $r$, by copying every spike from a common reference spike train with rate $r / c$ to any given target spike train with probability $c$ (Kuhn et al., 2003; Brette, 2009). Introducing very small correlations $(<1 \%)$ in this way also has a dramatic impact on the output firing rate of cortical cells (Fig. 13a,b-note the very small scale of the horizontal axis). The same phenomenon is seen in neuron models (Fig. 13c, left). These correlations are so small that they could probably not be seen in a paired recording: indeed, a correlation of $1 \%$ between two spike trains with rate $1 \mathrm{~Hz}$ results in one coincidence every $100 \mathrm{~s}$ on average.

This surprising sensitivity can be explained by the remark that the variance of the total synaptic input increases with input correlation, but a small pairwise correlation is still significant if there are many input pairs (approximately $N^{2}$ for $N$ synapses). More precisely, in a fluctuation-driven regime, the output rate critically depends on the input variance because the mean synaptic input is (by definition) below threshold. Suppose the total input consists of $N$ synaptic currents: $X=\Sigma I_{k}$, and these currents have variance $\sigma^{2}$ and pairwise correlation $c\left(c=\operatorname{covar}\left(I_{k}, I_{j}\right) / \sigma^{2}\right)$. If these currents were uncorrelated $(c=0)$, then the input variance would simply be $N \sigma^{2}$. When they are correlated, the input variance is the sum of the covariances all pairs of currents: $\operatorname{var}(X)=$ $\sum$ covar $\left(I_{k}, I_{j}\right)$. A simple calculation gives: $\operatorname{var}(X)=N \sigma^{2}+$ $c N(N-1) \sigma^{2}$. Thus, because there are $N(N-1)$ pairs of currents, the variance of the total input $X$ is mainly determined by the input correlations, unless $c$ is negligible compared with $1 / N$ (which is 
a

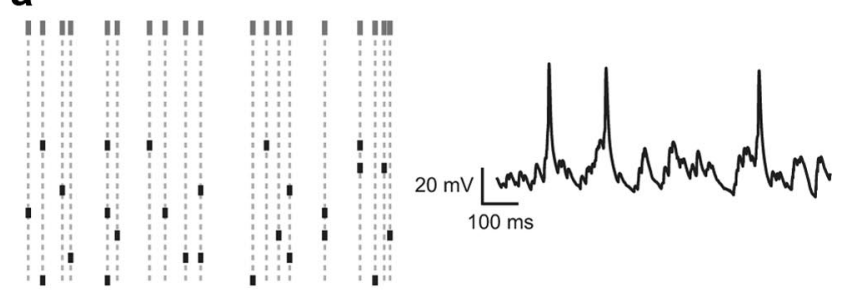

b
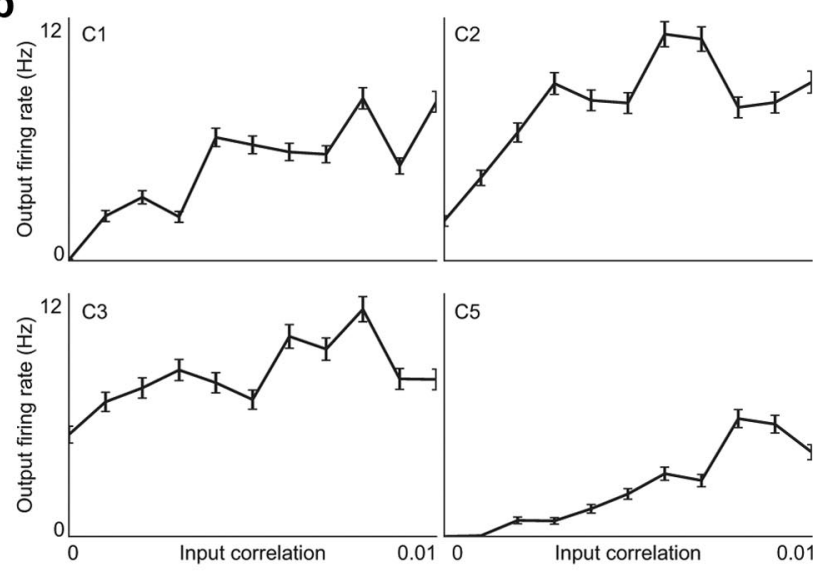

C
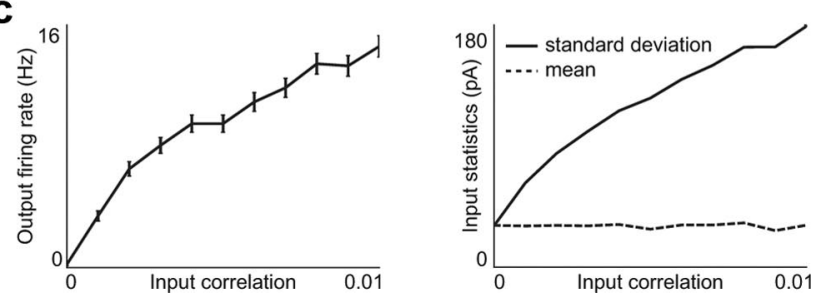

Figure 13. Impact of homogeneously correlated inputs on a cortical neuron. $\boldsymbol{a}, \mathrm{A}$ total of 4000 excitatory input spike trains with fixed pairwise correlation c are generated by thinning a common reference spike train (left), and injected into a cortical neuron with 1000 uncorrelated inhibitory spike trains (voltage response on the right). $\boldsymbol{b}$, Output firing rate of 4 cortical neurons as a function of the correlation coefficient $c$. $c$, The same correlated inputs are injected into an integrate-and-fire neuron model $\left(R=350 \mathrm{M} \Omega, \tau_{\mathrm{m}}=20 \mathrm{~ms}, E_{l}=-70 \mathrm{mV}, \theta=-45 \mathrm{mV}\right)$. Left, Output firing rate as a function of $c$. Right, Mean and SD of the total input current as a function of $c$.

already very small). This is shown in Figure $13 c$ (right), where the $\mathrm{SD}$ of the total current increases very quickly with correlation (note that the largest correlation considered is $c=1 \%$ ). Thus, very small pairwise correlations produce strong effects simply because there are many pairs, as was previously observed at network level in the retina (Schneidman et al., 2006).

\section{Transmission of correlations}

Precise coincidences increase the firing rate of a postsynaptic neuron, but is precise spike timing preserved in the operation? Figure $14 a$ shows a model example where the postsynaptic neuron fires at $2 \mathrm{~Hz}$ when the inputs are uncorrelated, and $8 \mathrm{~Hz}$ when synchrony is introduced $(p=10)$. Additional spikes caused by synchrony events are precisely time-locked to them, with each event triggering on average 0.16 extra spike (Fig. 14b). Therefore if several neurons receive independent inputs except for common synchrony events (involving $0.25 \%$ of synapses), then their firing is strongly correlated at a fine timescale (Fig. 14c). These correlations may then impact target postsynaptic neurons.

In Figure 14, output correlation is an order of magnitude larger than spike input correlation (between any two input spike trains), that is, spike correlation increases in the process. This may seem to contradict recent studies showing that the output correlation of a pair of neurons is always smaller than input correlation (de la Rocha et al., 2007; Shea-Brown et al., 2008), but it should be stressed that these studies compare the spike output correlation (correlation between two output spike trains) with the correlation between the two total synaptic currents. Total current correlation is essentially the sum of pairwise correlations (for all pairs of synaptic spike trains) and is therefore one order of magnitude larger (Renart et al., 2010; Rosenbaum et al., 2011). We illustrate this point in Figure 15, where we simulated two neurons with correlated input spike trains, with pairwise correlation $c$ (Fig. 15a). The correlation between the two total inputs $\left(c_{\text {current }}\right)$ is an order of magnitude larger than the spike input correlation $c$ (Fig. 15a, inset). This is a simple effect of pooling (Rosenbaum et al., 2011): the covariance between the two total inputs is the sum of the covariances of all pairs of synaptic currents, that is, $\mathrm{cN}^{2} \sigma^{2}$, and therefore the correlation is $c_{\text {current }}=$ $c N /(1+c(N-1))$. For a large number of synapses $N$, this is close to 1 , unless correlations are very small. As a result, the spike output correlation is much larger than the spike input correlation (Fig. 15b), even though it is smaller than the total current input correlation (Fig. 15c), in agreement with previous studies. In fact, the amplification of correlations is so strong that network stability requires that inhibition be correlated with excitation (Renart et al., 2010).

\section{Discussion}

\section{Sensitivity to fine correlations}

Our results show that neurons are highly sensitive to input correlations in the balanced (or fluctuation-driven) regime, when their timescale is smaller than the integration time constant. The required number of synchronous inputs for a strong effect is $\sim p \approx 10-20$ (assuming PSP sizes $\sim 1 \mathrm{mV}$, close to the average excitatory PSP size in the mouse auditory cortex in vitro (Oswald and Reyes, 2008); more generally: $p=$ (threshold - average $\left.V_{\mathrm{m}}\right)$ / PSP size). To have an idea of how strong this requirement is, let us consider the average number of excitatory input spikes within an integration window of $5 \mathrm{~ms}$ : with $N$ excitatory inputs at rate $F$, we obtain $F \times N \times 5$ ms. In Figure 10, for example $(F=1 \mathrm{~Hz}$ and $N=4000$ ), we obtain on average 20 spikes in one integration window. Therefore, a relatively small excursion above this average number has a very strong impact on postsynaptic firing. These results are consistent with recent findings that a small number $(20-40)$ of synchronous thalamic inputs can reliably drive cortical neurons (Bruno and Sakmann, 2006; Wang et al., 2010). In the latter study, authors found that spike efficacy was maximal for $\sim p=30$ synchronous inputs (that is, the ratio $P(p w) / p$, called reliability per synchrony magnitude, is maximal for $p=30$ ). Using parameter values from that study, our theoretical formula predicts a very similar value, $p=27$.

In this study, we defined the coincidence sensitivity as the difference in the average number of postsynaptic spikes (spike efficacy described by Usrey et al., 2000) produced by two synchronous input spikes versus two asynchronous spikes. Similar ideas were introduced by Abeles, who defined the "coincidence advantage" as the number of asynchronous spikes required to produce 0.5 average extra spike, divided by the number of synchronous spikes required to produce the same number of spikes (Abeles, 1982, 1991). The coincidence advantage corresponds to the ratio between the total colored area (blue + red) and the blue area in Figure $5 b$, that is, $P(p w) /(p P(w))$, where $p$ is the number of synchronous EPSPs required to produce 0.5 extra spike on aver- 
age (in our terms, this is approximately $p=(\theta-\mu) / w)$. Indeed, each asynchronous spike produces a postsynaptic spike with probability $P(w)$, and therefore $0.5 /$ $P(w)$ asynchronous spikes produce on average 0.5 postsynaptic spike. Thus the coincidence advantage is $0.5 /(p P(w))=$ $P(p w) /(p P(w))$ (colored area divided by blue area in Fig. $5 b$ ), for this particular value of $p$. This definition is related to the coincidence sensitivity $S_{p}$ for $p$ spikes (Fig. $8 c)$, which is $S_{p}=P(p w)-p P(w)$, except our definition is a difference while the coincidence advantage is a ratio. As we previously explained, it turned out that theoretical predictions worked better with a difference than with a ratio, because the errors in the estimation of $P(2 w)$ and $2 P(w)$ appear to approximately cancel (Fig. 5c).

In a recent theoretical study, it was found that a precise arrangement of excitation and inhibition could result in partial cancellation of correlations in a network, that is, pairwise correlations scale as $1 / N$ as the network size increases (Renart et al., 2010). In the light of our results, it should be stressed that this does not mean that correlations become negligible, in the sense that correlations of order $1 / N$ still modulate the output rate (correlations should be small compared with $1 / N$, e.g., $1 / N^{2}$, to be negligible). Indeed, the linear decrease in correlation is compensated by a linear increase in the number of synaptic inputs. From this point of view, describing the resulting network state as "asynchronous" may be misleading.

Fluctuation-driven versus mean-driven The main condition for neurons to be sensitive to coincidences is that the average input current is subthreshold, that is, that neurons are in a "fluctuation-driven" regime, as opposed to the "mean-driven" regime, where neurons fire regularly at a rate determined mainly by the mean current. This happens in particular when excitation balances inhibition on average, which occurs in vivo in the high-conductance regime induced by intense synaptic activity (Destexhe et al., 2003). Another signature of fluctuation-driven regimes is the temporal irregularity of spike trains. Recently, it was found that neurons in sensory cortices fire irregularly, while neurons in the motor cortex fire more regularly, but at a variable rate (Shinomoto et al., 2009). This would suggest that neurons in the sensory but not in the motor cortex are sensitive to coincidences. However, spike train regularity may also indicate strong oscillatory activity rather than a mean-driven regime, and therefore we cannot draw a firm conclusion from this observation. In vivo intracellular recordings in many areas (Fig. 8), including the primary motor cortex (Brecht et al., 2004), all indicate that the membrane potential distribution decreases toward spike threshold, which supports the notion that neurons are generally in a fluctuation-driven regime.

\section{Spike-timing versus rate in noisy neurons}

Our results support the view that synchrony plays an important role in neural computation. A central point in the debate between spike-timing and rate-based theories of neural computation is whether background activity should be treated as "noise" or "signal” (Stein et al., 2005; London et al., 2010)—that is, intertrial variability could reflect differences in network state rather than intrinsic noise. While our approach is agnostic about this point, our results show that, even if background activity reflects intrinsic or irreducible noise (e.g., chaos) (London et al., 2010), neurons are still extremely sensitive to the relative spike timing of their inputs. In other words, the presence of high voltage fluctuations implies that computation and coding are stochastic, but not that they are based on rate only. On the contrary, the fact that even very tiny correlations (of order $1 / N$ ) have tremendous postsynaptic impact suggests that neural computation is generally not satisfactorily described in terms of rates-whether synchrony is functionally useful or not.

\section{References}

Abeles M (1982) Role of the cortical neuron: integrator or coincidence detector? Isr J Med Sci 18:83-92.

Abeles M (1991) Corticonics: neural circuits of the cerebral cortex. Cambridge, UK: Cambridge UP. 
a

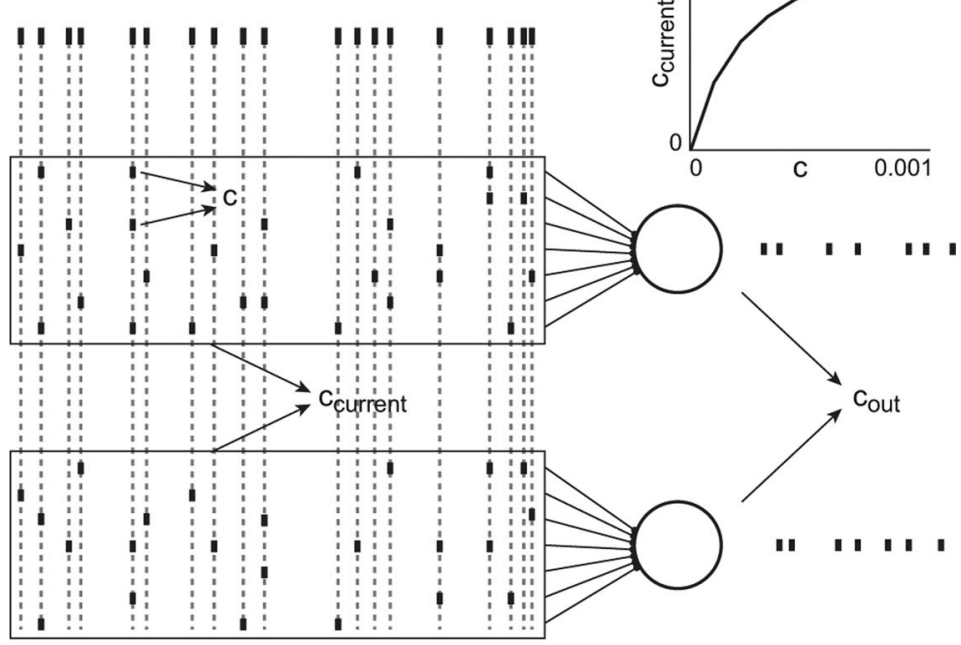

b

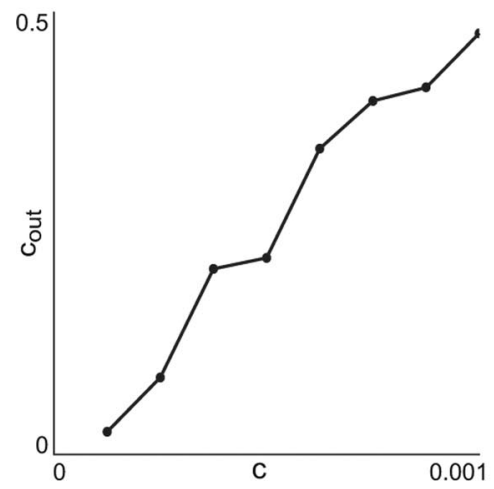

C

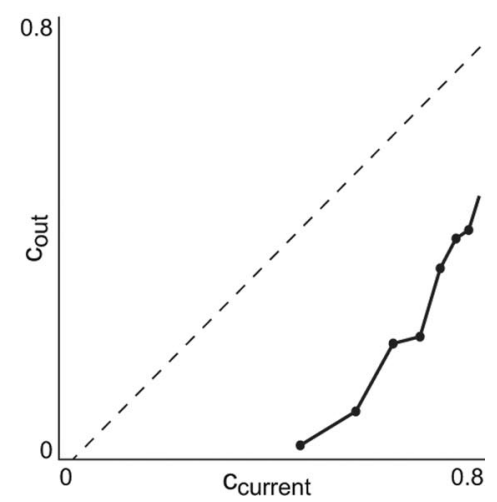

Figure 15. Transmission of correlations. $\boldsymbol{a}$, Two neurons receive correlated excitatory inputs as in Figure 3 (except inhibition is a constant current). The pairwise correlation between any two input spike trains is $c<0.001$, but the correlation $c_{\text {current }}$ between the two total currents is an order of magnitude larger. $\boldsymbol{b}$, As a result, output correlation is an order of magnitude larger than input correlation between single spike trains. $c$, However, it is smaller than input correlation between total currents, in agreement with previous findings.

Adrian ED (1934) The basis of sensation: the action of the sense organs. London: Christophers.

Alonso JM, Usrey WM, Reid RC (1996) Precisely correlated firing in cells of the lateral geniculate nucleus. Nature 383:815-819.

Azouz R, Gray CM (1999) Cellular mechanisms contributing to response variability of cortical neurons in vivo. J Neurosci 19:2209-2223.

Brecht M, Schneider M, Sakmann B, Margrie TW (2004) Whisker movements evoked by stimulation of single pyramidal cells in rat motor cortex. Nature 427:704-710.

Brette R (2009) Generation of correlated spike trains. Neural Comput 21:128-215.

Brette R, Piwkowska Z, Monier C, Rudolph-Lilith M, Fournier J, Levy M, Frégnac Y, Bal T, Destexhe A (2008) High-resolution intracellular recordings using a real-time computational model of the electrode. Neuron 59:379-391.

Brivanlou IH, Warland DK, Meister M (1998) Mechanisms of concerted firing among retinal ganglion cells. Neuron 20:527-539.

Bruno RM, Sakmann B (2006) Cortex is driven by weak but synchronously active thalamocortical synapses. Science 312:1622-1627.

Constantinople CM, Bruno RM (2011) Effects and mechanisms of wakefulness on local cortical networks. Neuron 69:1061-1068.

Crochet S, Petersen CC (2006) Correlating whisker behavior with membrane potential in barrel cortex of awake mice. Nat Neurosci 9:608-610.

de la Rocha J, Doiron B, Shea-Brown E, Josić K, Reyes A (2007) Correlation between neural spike trains increases with firing rate. Nature 448:802-806

Destexhe A, Rudolph M, Paré D (2003) The high-conductance state of neocortical neurons in vivo. Nat Rev Neurosci 4:739-751.

DeWeese MR, Zador AM (2006) Non-Gaussian membrane potential dynamics imply sparse, synchronous activity in auditory cortex. J Neurosci 26:12206-12218.

Fourcaud N, Brunel N (2002) Dynamics of the firing probability of noisy integrate-and-fire neurons. Neural Comput 14:2057-2110.

Gerstner W, Naud R (2009) How good are neuron models? Science 326:379-380.

Goodman DF, Brette R (2009) The brian simulator. Front Neurosci 3:192-197.

Goto Y, O'Donnell P (2001) Network synchrony in the nucleus accumbens in vivo. J Neurosci 21:4498-4504.

Harvey CD, Collman F, Dombeck DA, Tank DW (2009) Intracellular dynamics of hippocampal place cells during virtual navigation. $\mathrm{Na}$ ture 461:941-946.

Henze DA, Buzsáki G (2001) Action potential threshold of hippocampal pyramidal cells in vivo is increased by recent spiking activity. Neuroscience 105:121-130.

König P, Engel AK, Singer W (1996) Integrator or coincidence detector? The role of the cortical neuron revisited. Trends Neurosci 19:130-137.

Kuhn A, Aertsen A, Rotter S (2003) Higherorder statistics of input ensembles and the response of simple model neurons. Neural Comput 15:67-101.

Kumar A, Rotter S, Aertsen A (2010) Spiking activity propagation in neuronal networks: reconciling different perspectives on neural coding. Nat Rev Neurosci 11:615-627.

Léger JF, Stern EA, Aertsen A, Heck D (2005) Synaptic integration in rat frontal cortex shaped by network activity. J Neurophysiol 93:281-293.

London M, Roth A, Beeren L, Häusser M, Latham PE (2010) Sensitivity to perturbations in vivo implies high noise and suggests rate coding in cortex. Nature 466:123-127.

Meister M, Berry MJ (1999) The neural code of the retina. Neuron 22:435-450.

Moreno R, de la Rocha J, Renart A, Parga N (2002) Response of spiking neurons to correlated inputs. Phys Rev Lett 89:288101.

Oswald AM, Reyes AD (2008) Maturation of intrinsic and synaptic properties of layer $2 / 3$ pyramidal neurons in mouse auditory cortex. J Neurophysiol 99:2998-3008.

Renart A, de la Rocha J, Bartho P, Hollender L, Parga N, Reyes A, Harris KD (2010) The asynchronous state in cortical circuits. Science 327:587-590.

Richardson MJ, Gerstner W (2005) Synaptic shot noise and conductance fluctuations affect the membrane voltage with equal significance. Neural Comput 17:923-947.

Richardson MJ, Gerstner W (2006) Statistics of subthreshold neuronal voltage fluctuations due to conductance-based synaptic shot noise. Chaos 16:026106.

Richardson MJ, Swarbrick R (2010) Firing-rate response of a neuron receiving excitatory and inhibitory synaptic shot noise. Phys Rev Lett 105:178102

Rosenbaum R, Trousdale J, Josić K (2011) The effects of pooling on correlated neural variability. Front Neurosci 5:58.

Rossant C, Goodman DF, Fontaine B, Platkiewicz J, Magnusson AK, Brette R (2011) Fitting neuron models to spike trains. Front Neurosci 5:9.

Roy SA, Alloway KD (2001) Coincidence detection or temporal integration? What the neurons in somatosensory cortex are doing. J Neurosci 21:2462-2473.

Rudolph M, Destexhe A (2003a Characterization of subthreshold voltage fluctuations in neuronal membranes. Neural Comput 15:2577-2618.

Rudolph M, Destexhe A (2003b) Tuning neocortical pyramidal neurons be- 
tween integrators and coincidence detectors. J Comput Neurosci 14:239-251.

Salinas E, Sejnowski TJ (2000) Impact of correlated synaptic input on output firing rate and variability in simple neuronal models. J Neurosci 20:6193-6209.

Salinas E, Sejnowski TJ (2001) Correlated neuronal activity and the flow of neural information. Nat Rev Neurosci 2:539-550.

Schneidman E, Berry MJ 2nd, Segev R, Bialek W (2006) Weak pairwise correlations imply strongly correlated network states in a neural population. Nature 440:1007-1012.

Shadlen MN, Newsome WT (1998) The variable discharge of cortical neurons: implications for connectivity, computation, and information coding. J Neurosci 18:3870-3896.

Shea-Brown E, Josić K, de la Rocha J, Doiron B (2008) Correlation and synchrony transfer in integrate-and-fire neurons: basic properties and consequences for coding. Phys Rev Lett 100:108102.

Shinomoto S, Kim H, Shimokawa T, Matsuno N, Funahashi S, Shima K, Fujita I, Tamura H, Doi T, Kawano K, Inaba N, Fukushima K, Kurkin S, Kurata K, Taira M, Tsutsui K, Komatsu H, Ogawa T, Koida K, Tanji J, Toyama K (2009) Relating neuronal firing patterns to functional differentiation of cerebral cortex. PLoS Comput Biol 5:e1000433.

Singer W (1999) Time as coding space? Curr Opin Neurobiol 9:189-194.

Softky WR, Koch C (1993) The highly irregular firing of cortical cells is inconsistent with temporal integration of random EPSPs. J Neurosci 13:334-350.

Stein RB, Gossen ER, Jones KE (2005) Neuronal variability: noise or part of the signal? Nat Rev Neurosci 6:389-397.

Stern EA, Kincaid AE, Wilson CJ (1997) Spontaneous subthreshold membrane potential fluctuations and action potential variability of rat corticostriatal and striatal neurons in vivo. J Neurophysiol 77:1697-1715.

Stopfer M, Bhagavan S, Smith BH, Laurent G (1997) Impaired odour discrimination on desynchronization of odour-encoding neural assemblies. Nature 390:70-74

Usrey WM, Reid RC (1999) Synchronous activity in the visual system. Annu Rev Physiol 61:435-456.

Usrey WM, Reppas JB, Reid RC (1998) Paired-spike interactions and synaptic efficacy of retinal inputs to the thalamus. Nature 395:384-387.

Usrey WM, Alonso JM, Reid RC (2000) Synaptic interactions between thalamic inputs to simple cells in cat visual cortex. J Neurosci 20:5461-5467.

VanRullen R, Guyonneau R, Thorpe SJ (2005) Spike times make sense. Trends Neurosci 28:1-4.

Wang HP, Spencer D, Fellous JM, Sejnowski TJ (2010) Synchrony of thalamocortical inputs maximizes cortical reliability. Science 328:106109.

Wilson CJ, Kawaguchi Y (1996) The origins of two-state spontaneous membrane potential fluctuations of neostriatal spiny neurons. J Neurosci 16: 2397-2410. 